\title{
CaMK4 compromises podocyte function in autoimmune and nonautoimmune kidney disease
}

\author{
Kayaho Maeda, ${ }^{1}$ Kotaro Otomo, ${ }^{1}$ Nobuya Yoshida, ${ }^{1}$ Mones S. Abu-Asab, ${ }^{2}$ Kunihiro Ichinose, ${ }^{3}$ Tomoya Nishino, ${ }^{4}$ Michihito Kono, ${ }^{1}$ \\ Andrew Ferretti, ${ }^{1}$ Rhea Bhargava, ${ }^{1}$ Shoichi Maruyama, ${ }^{5}$ Sean Bickerton, ${ }^{6}$ Tarek M. Fahmy, ${ }^{7}$ Maria G. Tsokos, ${ }^{1}$ and George C. Tsokos ${ }^{1}$ \\ 'Department of Medicine, Beth Israel Deaconess Medical Center, Harvard Medical School, Boston, Massachusetts, USA. ${ }^{2}$ Histopathology Core, National Eye Institute, NIH, Bethesda, Maryland, USA. \\ ${ }^{3}$ Department of Immunology and Rheumatology, Division of Advanced Preventive Medical Sciences, Nagasaki University Graduate School of Biomedical Sciences, Nagasaki, Japan. ${ }^{2}$ Department of \\ Nephrology, Nagasaki University Hospital, Nagasaki, Japan. ${ }^{5}$ Department of Nephrology, Nagoya University Graduate School of Medicine, Nagoya, Japan. ${ }^{6}$ Department of Biomedical Engineering, Yale \\ University and Yale University School of Medicine, New Haven, Connecticut, USA. 'Department of Biomedical Engineering, Department of Chemical and Environmental Engineering, Department of \\ Immunobiology, Yale University and Yale University School of Medicine, New Haven, Connecticut, USA.
}

\begin{abstract}
Podocyte malfunction occurs in autoimmune and nonautoimmune kidney disease. Calcium signaling is essential for podocyte injury, but the role of $\mathrm{Ca}^{2+} / c^{2}$ lmodulin-dependent kinase (CaMK) signaling in podocytes has not been fully explored. We report that podocytes from patients with lupus nephritis and focal segmental glomerulosclerosis and lupusprone and lipopolysaccharide- or adriamycin-treated mice display increased expression of CaMK IV (CaMK4), but not CaMK2. Mechanistically, CaMK4 modulated podocyte motility by altering the expression of the GTPases Rac1 and RhoA and suppressed the expression of nephrin, synaptopodin, and actin fibers in podocytes. In addition, it phosphorylated the scaffold protein 14-3-3 $\beta$, which resulted in the release and degradation of synaptopodin. Targeted delivery of a CaMK4 inhibitor to podocytes preserved their ultrastructure, averted immune complex deposition and crescent formation, and suppressed proteinuria in lupus-prone mice and proteinuria in mice exposed to lipopolysaccharide-induced podocyte injury by preserving nephrin/synaptopodin expression. In animals exposed to adriamycin, podocyte-specific delivery of a CaMK4 inhibitor prevented and reversed podocyte injury and renal disease. We conclude that CaMK4 is pivotal in immune and nonimmune podocyte injury and that its targeted cell-specific inhibition preserves podocyte structure and function and should have therapeutic value in lupus nephritis and podocytopathies, including focal segmental glomerulosclerosis.
\end{abstract}

\section{Introduction}

Podocyte dysfunction is a common feature of renal injury in autoimmune and nonautoimmune renal diseases, including lupus nephritis (LN) and focal segmental glomerulosclerosis (FSGS). Sustained podocyte injury ultimately leads to chronic kidney disease (CKD). At present, progressive kidney disease is not curable with available treatment strategies $(1,2)$. Podocytes are unique cells on the outer aspect of the glomerular capillaries that form interdigitating foot processes connected with a highly specialized junction, the slit diaphragm. The actin-rich foot processes and the slit diaphragm comprise a complex of proteins, including nephrin and podocin, and constitute the main glomerular filtration barrier (3). Actin fiber remodeling and cytoskeletal rearrangement lead to foot process effacement, slit diaphragm disruption, and podocyte detachment $(4,5)$. Calcium $\left(\mathrm{Ca}^{2+}\right)$ signaling plays a critical role in podocyte injury. Increases of $\mathrm{Ca}^{2+}$ in podocytes lead to degradation of synaptopodin, an actin-binding protein involved in the regulation of podocyte integrity, and modulation of the signaling by the Rho family of small GTPases, including Rac1, Cdc42, and RhoA, which control cytoskeletal dynamics and the shape and motility of

Authorship note: KM and $\mathrm{KO}$ contributed equally to this work.

Conflict of interest: The authors have declared that no conflict of interest exists.

Submitted: December 27, 2017; Accepted: May 17, 2018.

Reference information: J Clin Invest. 2018;128(8)3445-3459.

https://doi.org/10.1172/JCI99507. the foot processes $(6,7)$. Synaptopodin is dephosphorylated by the $\mathrm{Ca}^{2+}$-calmodulin-dependent phosphatase calcineurin, which allows its release from the chaperone 14-3-3 $\beta$ protein and its subsequent proteolytic degradation. On the other hand, synaptopodin is phosphorylated and stabilized by $\mathrm{Ca}^{2+} / \mathrm{calmodulin}$-dependent kinase II (CaMK2) (8), but CaMK2 inhibition has mitigated angiotensin IItriggered podocyte injury and proteinuria in mice (9).

The $\mathrm{Ca}^{2+}$ /calmodulin-dependent protein kinase IV (CaMK4), another member of the CaMK family, is a multifunctional serine/ threonine kinase that regulates several aspects of the immune response (10). CaMK4 is increased in the nuclei of $\mathrm{T}$ cells from patients with systemic lupus erythematosus (SLE) and activates the repressor cAMP response element modulator $\alpha$ and the AKT/mammalian target of rapamycin pathway (11), leading to decreased IL-2 and increased IL-17 production. Pharmacologic inhibition (12) or genetic deletion of CaMK4 (13) and targeted delivery of a CaMK4 inhibitor to $\mathrm{CD}^{+}{ }^{+} \mathrm{T}$ cells (14) effectively prevented LN in the lupus-prone MRL.lpr mouse. We have found that CaMK4 was increased and colocalized with nephrin in the glomeruli of SLE patients and that IgG from LN patients upregulated CaMK4 in podocyte cultures (15).

Here, we report that CaMK4 is directly involved in podocyte injury of diverse etiology because it is upregulated, not only in autoimmune, but also in nonautoimmune, podocytopathies in humans and mice and interferes with cytoskeletal dynamics and slit diaphragm function by downregulating the expression 
of nephrin and synaptopodin. We further reveal a mechanism whereby CaMK4 affects podocyte motility through the activation of Rac1 and phosphorylation of the scaffold protein 14-3-3, which leads to release and degradation of synaptopodin. More importantly, we show that targeted delivery of the CaMK4 inhibitor to podocytes preserves their structure, prevents the development of glomerulonephritis in lupus-prone mice, and reverses adriamycininduced podocyte injury in mice. Our studies introduce CaMK4 as a common pathogenetic node of diverse podocytopathies and as a therapeutic target for mitigating podocyte injury.

\section{Results}

CaMK4 is upregulated in podocytes in autoimmune and nonautoimmune kidney disease. We had previously observed that CaMK4 is increased in the podocytes of patients with LN (15). To determine whether CaMK4 has a role in nonautoimmune kidney disease, we studied the expression of CaMK4 in renal biopsies from patients with FSGS, which is characterized by primary nonautoimmune podocyte injury. We found CaMK4 expression to be increased in the glomeruli of patients with FSGS at levels comparable to those noted in SLE patients. Furthermore, CaMK4 was colocalized with nephrin, consistent with a podocyte source (Figure 1A and Supplemental Figure 1A; supplemental material available online with this article; https://doi.org/10.1172/ JCI99507DS1). Interestingly, nephrin appeared to be decreased in the CaMK4-overexpressing glomeruli from both SLE and FSGS kidneys when compared with normal kidneys (Figure 1A). Main clinical features of the study subjects are provided in Supplemental Table 1. Unlike CaMK4, CaMK2 expression in the kidney biopsies from SLE and FSGS patients was comparable to levels observed in normal kidney tissue (Figure 1B). We exposed human podocytes cultured in vitro to IgG from lupus patients or to lipopolysaccharide (LPS), and we noted upregulation of CaMK4 expression, while the levels of CaMK2 did not change (Figure 1, C-F, and Supplemental Figure 1B). Taking these data together, it appears that CaMK4, but not CaMK2, is linked to podocyte injury under these experimental conditions.

To study further the role of CaMK4 in podocytopathies, we created conditions of experimental podocyte injury by injecting mice with LPS or adriamycin, which are known to damage podocytes, leading to proteinuria. We found increased expression of CaMK4 in the glomeruli of both LPS- and adriamycin-treated mice when compared with control mice. We also noted coexpression of CaMK4 with the podocyte-specific protein synaptopodin, which was expressed at significantly lower levels than in control mice (Figure 2A), suggesting a reverse association with CaMK4, similar to the one we noted with nephrin levels in the human kidney biopsies. CaMK4 was not detected in Camk4-deficient mice (Supplemental Figure 2A). These observations, i.e., increased glomerular expression of CaMK4 and decreased expression of synaptopodin, were corroborated with findings in the kidneys of lupusprone MRL.lpr mice. We observed that CaMK4 levels increased in podocytes of MRL.lpr mice, as compared with MRL/MpJ control mice; these increased levels first appeared at week 12, and levels increased further at 16 weeks of age, coinciding with the time that the mice developed proteinuria (Figure 2B). Consistent with these data, CaMK4 mRNA levels also increased in isolated glomeruli and, more importantly, in flow cytometry-sorted podocytes from the diseased mice compared with control mice (Figure 2, C-F, and Supplemental Figure 3). CaMK4 was not detected in sorted podocytes from Camk4-deficient mice (Supplemental Figure 2B). These data indicate that CaMK4 represents a common node in the expression of podocyte injury in patients and mice with immune and nonimmune podocyte injury. The age-dependent appearance of increased CaMK4 expression in lupus-prone mice points to its clinical value for detecting early podocyte injury.

Podocyte-specific delivery of a CaMK4 inhibitor averts LN in mice. Prior to delving into the mechanisms whereby CaMK4 suppresses the expression of nephrin and synaptopodin, we wished to determine whether inhibition of CaMK4 in a podocyte-specific manner can preserve podocyte function and prevent kidney disease in lupus-prone mice. To accomplish this, we prepared nanolipogels (nlg) tagged with antibodies to podocin or nephrin, which we found to be readily detected by flow cytometry on the surface of podocytes maturing in culture (16) (Supplemental Figure 4). First, we injected rhodamine-labeled nlg (17) tagged with anti-podocin or anti-nephrin into 12-week-old MRL.lpr mice and documented that they localized extensively in the glomeruli 30 minutes after injection, whereas control, non-antibody-tagged nlg were found mainly outside of the glomeruli (Supplemental Figure 5). To assess the efficacy of anti-podocin- or anti-nephrin-tagged nlg loaded with the CaMK4 inhibitor KN93 in preventing the development of LN in lupus-prone mice, we injected nlg i.p. weekly in MRL.lpr mice beginning at 8 weeks of age. The treatment did not affect skin lesions or the weight of spleens and kidneys at 16 weeks of age (Supplemental Figure 6). In addition, dsDNA antibody titers and serum IL-17 levels were not affected, although IL-2 levels increased (Supplemental Figure 7, A-C). The numbers of regulatory $\mathrm{T}$ cells in the spleen or lymph nodes were not affected (Supplemental Figure 7D). Targeted delivery of KN93 with antipodocin- or anti-nephrin-tagged nlg had a profound effect on proteinuria compared with what occurred in animals treated with an equivalent amount of free KN93 (Figure 3A). Treatment of MRL.lpr mice with anti-nephrin-coated KN93-loaded nlg prevented the development of nephritic histopathology (Figure 3 , B-D) and podocyte foot process effacement, as documented by electron microscopy (Figure 3, E and F). Blood urea nitrogen (BUN) levels from these mice also reflected the effect of antinephrin-coated nlg loaded with KN93 (Supplemental Figure 7E). Interestingly, we noted decreased deposition of C3 and IgG in the glomeruli of treated mice (Figure $3 G$ ) and none to very few dense deposits in the subepithelial and mesangial areas of the treated mice as opposed to the abundant dense deposits that were present in the nontreated mice (Figure 3, C, E, and F). Preservation of podocyte structure was associated with high expression of nephrin and synaptopodin proteins, which regulate actin-based shape and motility of podocyte foot processes, as evaluated by confocal microscopy (Figure 3, H and I). Similar results were obtained when we quantified the expression of Nphs1 (encoding for nephrin), Nphs2 (encoding for podocin), and Synpo (encoding for synaptopodin) mRNA in glomeruli from mice treated with antinephrin-coated KN93-loaded nlg or control (empty) nlg, pointing to a transcriptional control in their expression (Figure 3J). Similarly, crescent formation was not observed in the glomeruli of treated 
A

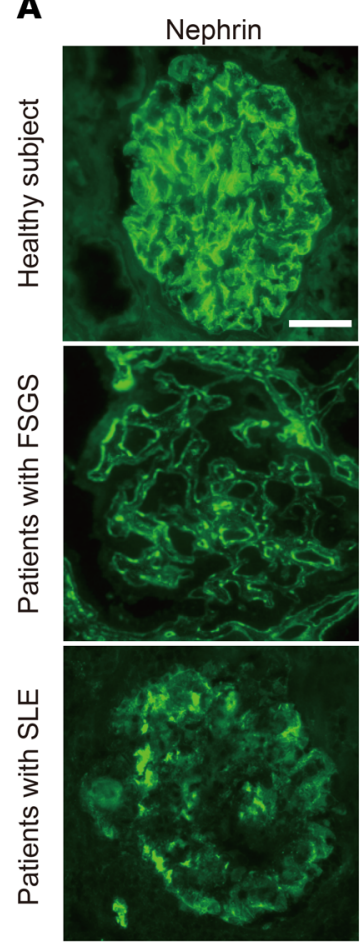

B
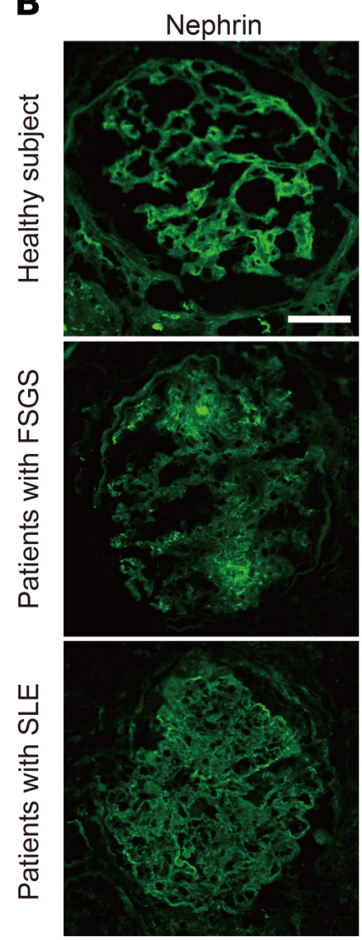

CaMK4
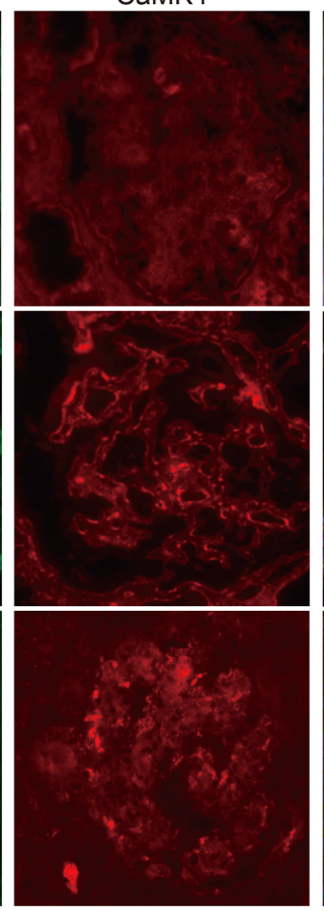

CaMK2
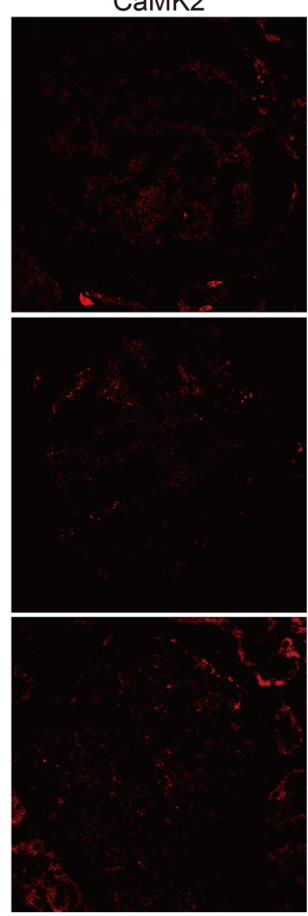

Merge
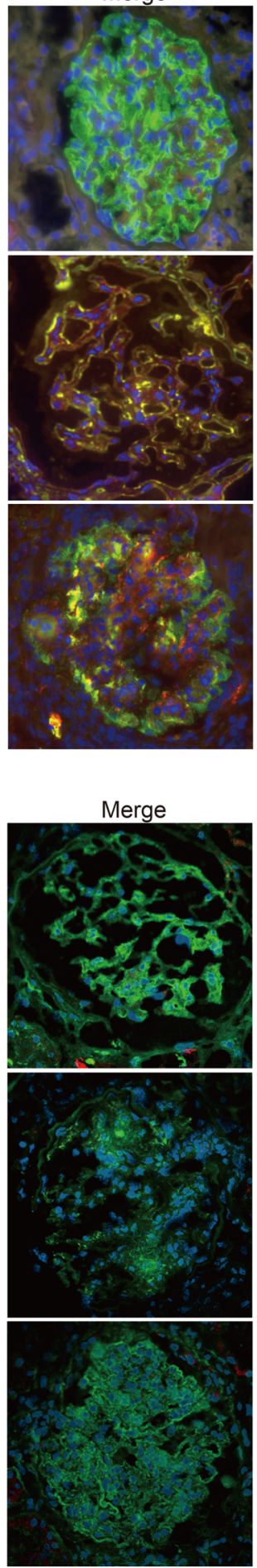

C

CaMK

GAPDH

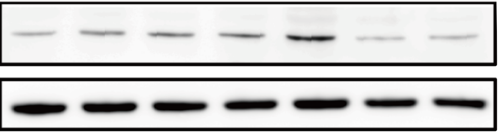

$\begin{array}{llllllll}0 & \mathrm{~N} 24 & \mathrm{~L} 24 & \mathrm{~N} 48 & \mathrm{~L} 48 & \mathrm{~N} 72 & \mathrm{~L} 72 & (\mathrm{~h})\end{array}$

\section{D}

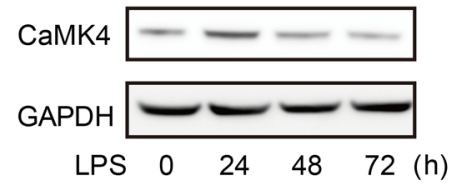

E

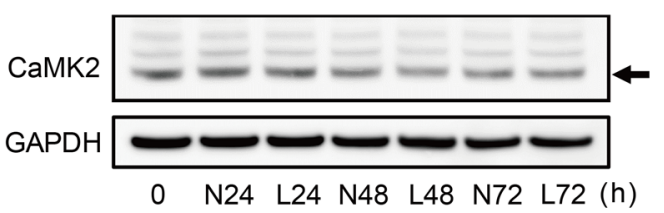

$\mathbf{F}$

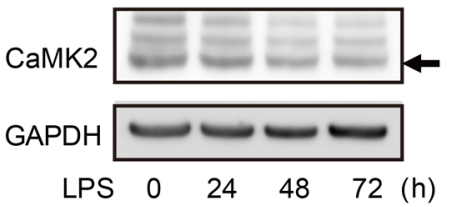

Figure 1. CaMK4 is upregulated in podocytes from autoimmune/nonautoimmune kidney diseases. (A) Representative images of immunofluorescence for nephrin or CaMK4 from patients with $\mathrm{LN}(n=10)$ or FSCS $(n=10)$ or from a subject without renal disease. Green or red color represents nephrin or CaMK4, respectively, and merged figure shows the signals from nephrin, CaMK4, and DAPI staining. Scale bar: $50 \mu \mathrm{m}$. (B) Representative images of immunofluorescence for nephrin or CaMK2 from patients with LN $(n=10)$ or FSCS $(n=10)$ or from a subject without renal disease. Green or red color represents nephrin or CaMK2, respectively, and the merged figure shows the signals from nephrin, CaMK2, and DAPI staining. Scale bar: $50 \mu \mathrm{m}$. (C and D) Time course of CaMK4 expression in human podocytes after exposure to IgG from LN patients (L) or healthy donors (N) (C) or LPS (D). The data are representative of 3 independent experiments. (E and $\mathbf{F}$ ) Time course of CaMK2 (arrow) expression in human podocytes after exposure to IgG from LN patients or healthy donors (E) or LPS (F). The data are representative of 3 independent experiments. mice (Figure 3B), an observation which is consistent with the fact that injured podocytes contribute to the development of crescents (18). These data suggest that CaMK4 upregulation is required for and precedes the development of podocyte injury, crescent formation, and immune complex deposition. The absolute numbers of lymphocytes (Supplemental Figure 8A) and the numbers of IFN- $\gamma^{+}$-producing $\mathrm{CD}^{+}$cells (Supplemental Figure $8 \mathrm{~B}$ ) and those of $\mathrm{CD}^{2} 5^{+} \mathrm{FOXP}^{+} \mathrm{CD} 4^{+} \mathrm{T}$ cells (Supplemental Figure $8 \mathrm{C}$ ) infiltrating the kidneys of MRL.lpr mice treated with podocytedirected KN93-loaded nlg did not differ from those of the control-treated mice.

Inhibition of CaMK4 prevents LPS-induced podocyte injury. Because delivery of KN93 to podocytes suppressed proteinuria in lupus-prone mice and because CaMK4 was increased in mice injected with LPS or adriamycin, we asked whether similar treatment would suppress proteinuria in nonimmune injury of podo- 
A

Synaptopodin
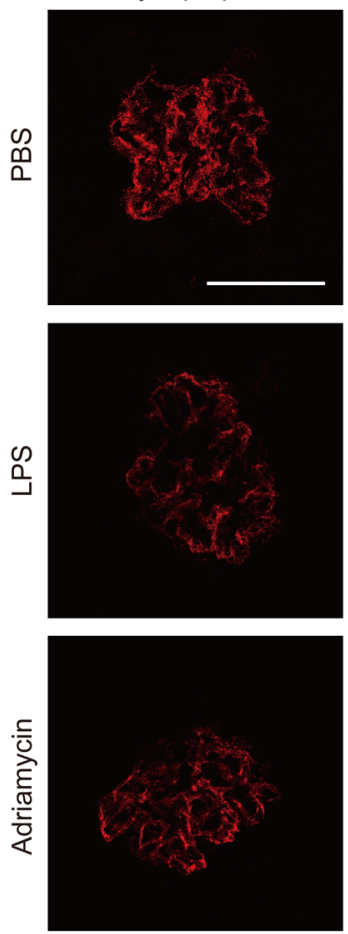

B
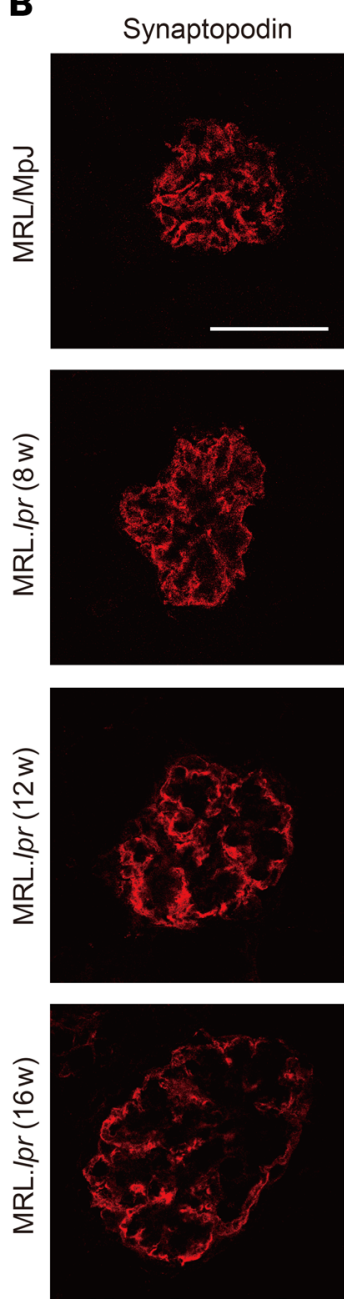

CaMK4
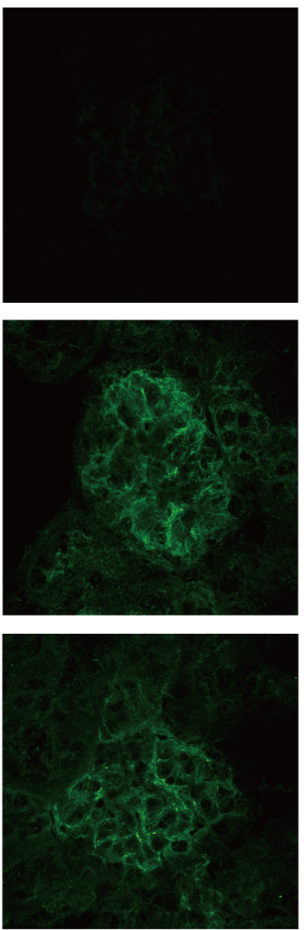

CaMK4
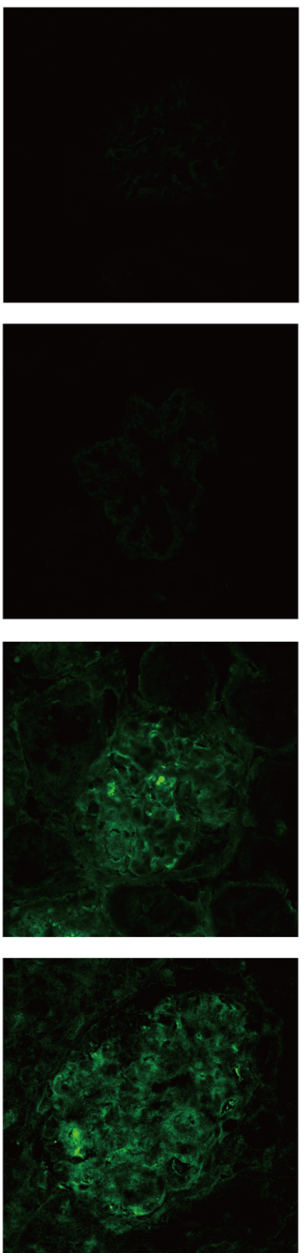

Merge
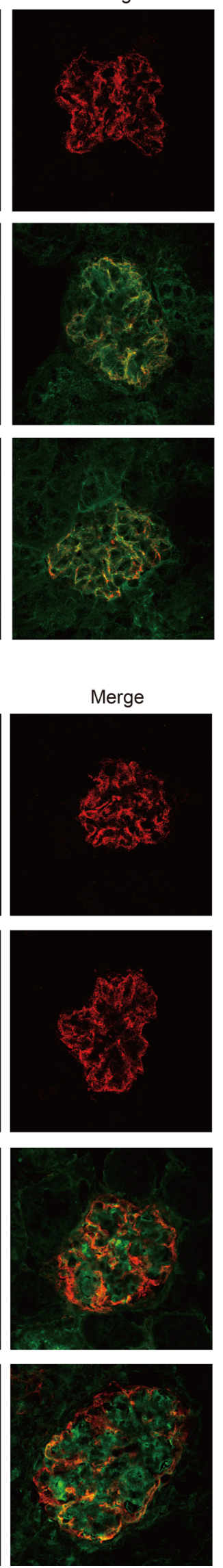

C
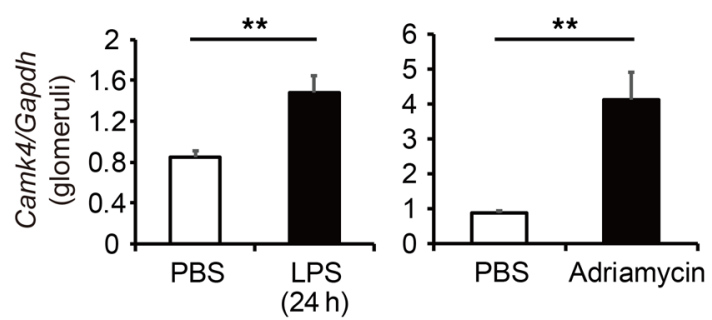

D
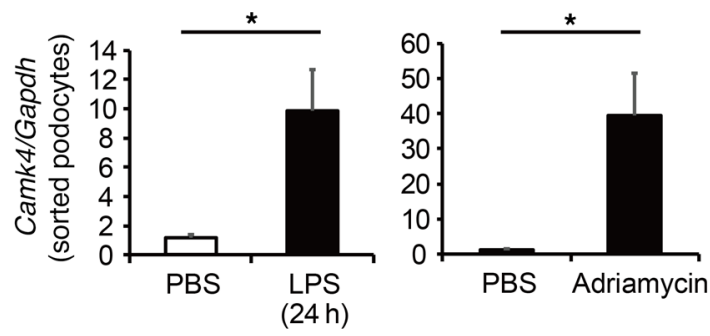

$\mathbf{E}$

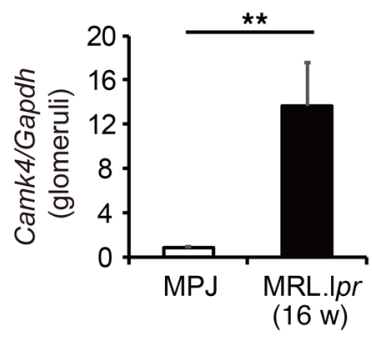

$\mathbf{F}$

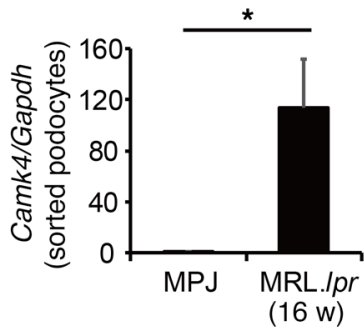

Figure 2. CaMK4 expression is increased in podocytes from lupus-prone and LPS- or adriamycin-treated mice. (A) Representative images of immunofluorescence for synaptopodin or CaMK4 in PBS-, LPS-, and adriamycin-treated mice. Scale bar: $50 \mu \mathrm{m}$. (B) Representative images of immunofluorescence for synaptopodin or CaMK4 in MRL/MpJ and MRL.Ipr mice $(8,12,16$ weeks old). Scale bar: $50 \mu \mathrm{m}$. (C-F) Camk4 mRNA expression in glomeruli (C and $\mathbf{E}$ ) or sorted podocytes by flow cytometry ( $\mathbf{D}$ and F) from PBS-, LPS-, and adriamycin- treated mice and MRL/MpJ and MRL.Ipr mice (16 weeks old). Results were normalized to the expression of GAPDH ( $n=5$ in each group). ${ }^{*} P<0.05$; ${ }^{*} P<0.01$, Student's $t$ test. 
cytes. LPS injection resulted in proteinuria within 24 hours in WT mice (19), but significantly less proteinuria was observed in mice treated with anti-nephrin-tagged KN93-loaded nlg or in $\mathrm{Camk}^{-/-}$mice (Figure 4, A and B). Because nephrin and synaptopodin are essential to the function and structure of podocytes and were downregulated in lupus-prone mice and patients with LN and FSGS in conjunction with CaMK4 upregulation, we asked whether nephrin and synaptopodin in LPS-treated mice are also downregulated in a CaMK4-dependent manner. Indeed, immunofluorescence studies revealed that exposure of WT, but not $\mathrm{Camk4}^{-/}$, mice to LPS resulted in decreased expression of nephrin (Figure 4C). Similarly, synaptopodin expression was preserved in Camk4 ${ }^{-/}$, but not in WT, mice treated with LPS (Figure 4C and Supplemental Figure 2C). We extended these findings in cultured human podocytes and found that CaMK4 was also upregulated after exposure to LPS (Figure 1D and Supplemental Figure 1B) and that the presence of KN93 preserved the expression of nephrin mRNA and protein (Figure 4, D and E, and Supplemental Figure 9). Finally, silencing of CaMK4 with siRNA also preserved nephrin expression (Figure $4 \mathrm{~F}$ ). These data reveal that induction of CaMK4 by LPS leads to suppression of nephrin and synaptopodin, leading to albuminuria, whereas its genetic absence or pharmacologic inhibition preserves the expression of these proteins and averts proteinuria.

Podocyte-targeted KN93 is effective in protecting against adriamycin-induced podocytopathy. Administration of adriamycin to mice causes severe proteinuria as early as within 1 week (20), and it has served as a tool to study aspects of FSGS. To determine whether adriamycin-induced podocyte injury involves upregulation of CaMK4 (Figure 2A) in a manner similar to that noted in the case of LPS, we injected mice with anti-nephrin-tagged KN93-loaded nlg i.p. once a day prior to the administration of adriamycin. Such treatment suppressed proteinuria (Figure 5A), and 7 days after the administration of adriamycin, renal histopathology showed hyaline deposits in glomeruli and tubulointerstitial damage with intratubular casts in the mice treated with control nlg, but not in mice treated with KN93-loaded nlg tagged with anti-nephrin antibody (Figure 5B). Similar to LPS, injection of adriamycin suppressed the expression of nephrin and synaptopodin, as documented in immunofluorescence studies, whereas treatment with KN93-loaded nlg tagged with anti-nephrin antibody preserved their expression (Figure 5C). To address whether adriamycin-induced kidney disease is reversible by CaMK4 inhibitors, we started daily administration of anti-nephrin-tagged KN93-loaded nlg 7 days after the injection of adriamycin when overt proteinuria had appeared.

Podocyte-targeted delivery of KN93 dramatically reduced proteinuria at day 14 compared with control nlg or an equivalent dose of KN93 administered systemically (Figure 5D). Consistent with these results, CaMK4-deficient mice treated with adriamycin displayed limited proteinuria (Figure 5E). Electron microscopy studies revealed that inhibition of CaMK4 in a targeted manner limited podocyte injury (Figure $5 \mathrm{~F}$ ). These findings demonstrate that adriamycin involves the upregulation of CaMK4 and the subsequent suppression of the expression of nephrin and synaptopodin to cause renal damage and proteinuria. The ability of CaMK4 inhibitors to prevent and reverse ongoing glomerular damage carries substantial translational value.
CaMK4 induces Rac-1 activation and regulates the motility of human podocytes. EM studies reported above in lupus-prone MRL.lpr and adriamycin-treated mice showed that CaMK4 inhibition averted podocyte foot process effacement. To address how CaMK4 promotes foot process effacement, we examined the motility of podocytes because motile imbalance promotes foot process effacement and disrupts the podocyte slit diaphragm (21, 22). To investigate the role of CaMK4 in the motility of podocytes, we analyzed cell migration of podocytes in which CaMK4 had been silenced. First, using a Transwell system with type I collagencoated membranes, we found that podocytes in which CaMK4 had been silenced were less motile than control cells (Figure 6A). We expanded the study of podocyte migration by using a woundhealing assay and calculated the number of cells migrating into the scratched area. Silencing of CaMK4 significantly inhibited directional podocyte movement to fill the wound in contrast with what occurred in control cells (Figure 6, B and C). Small GTPases, such as RhoA, Rac1, and Cdc42, regulate actin cytoskeletal dynamics and podocyte shape change. Rac1 and Cdc42 promote cell motility through the formation of lamellipodia and filopodia. Conversely, RhoA is implicated in a contractile phenotype through stress fiber formation $(23,24)$. Alterations in the balance of small GTPases lead to hypo- or hypermotility of podocytes, which causes proteinuria $(22,25,26)$. We found that GTP-bound activated Rac1 increased, while GTP-bound activated RhoA decreased within 15 minutes of podocyte exposure to LPS and that both were reversed after CaMK4 silencing (Figure 6, D and E). These data indicate that increased CaMK4 levels promote podocyte motility by regulating the ratio of activated Rac1 and RhoA GTPases.

Inhibition of CaMK4 preserves actin fibers and LPS-induced synaptopodin degradation in podocytes. Continuous $\mathrm{Ca}^{2+}$ influx and signaling are associated with podocyte motility and actin fiber damage $(6,27)$. Inhibition of the canonical transient receptor potential channels 5 (TRPC5), one of the $\mathrm{Ca}^{2+}$ channels on podocytes, abrogates podocyte cytoskeletal remodeling that leads to proteinuria (7). Since CaMK4 is $\mathrm{Ca}^{2+}$ dependent, we silenced TRPC5 in podocytes and found that the LPS-induced upregulation of CaMK4 was abrogated (Supplemental Figure 10, A-C). It has been reported that inhibition or genetic deficiency of TRPC5 prevents the activation of the small GTP-binding protein Rac1 and stabilizes synaptopodin (7). Therefore, we considered that inhibition of CaMK4 could protect actin fibers and cytoskeleton structure by stabilizing synaptopodin. Indeed, we observed that KN93 treatment (Figure 7, A and B) or silencing of CaMK4 (Figure 7, C and D) protected podocytes from cytoskeletal damage and preserved the structure of actin fibers. Synaptopodin is expressed in podocytes and orchestrates the organization of actin cytoskeleton by regulating $\alpha$-actinin 4 activity and small GTPases $(8,23)$. Pharmacological inhibition of CaMK4 protected synaptopodin from LPS-induced degradation in a dose-dependent manner (Figure 8, A and B), and silencing of CaMK4 similarly preserved the expression of synaptopodin (Figure 8, C and D). Since synaptopodin knockdown in podocytes results in impaired motility (23) and LPS-induced suppression of synaptopodin expression was preserved by CaMK4 inhibition, we evaluated podocyte motility after exposure to LPS and noted that it was decreased. This decrease was averted when CaMK4 was silenced (Supplemental 
A

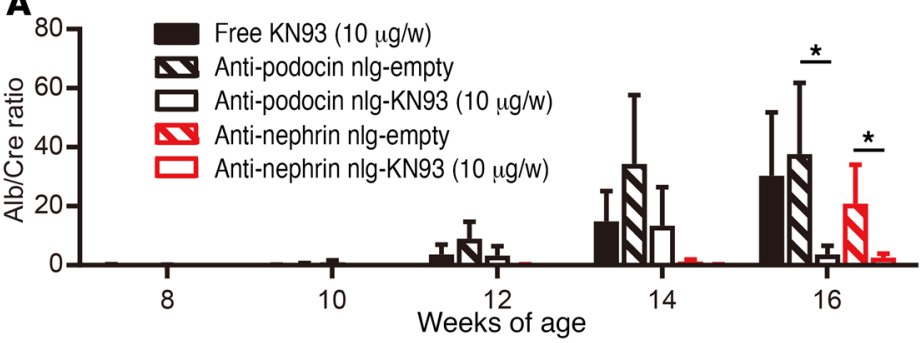

B

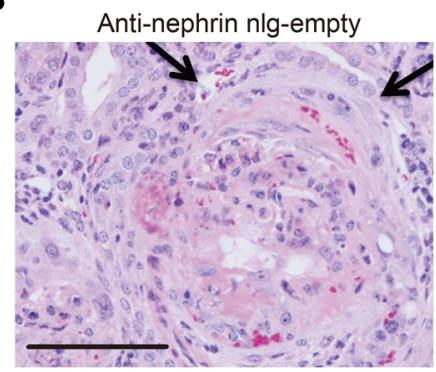

C
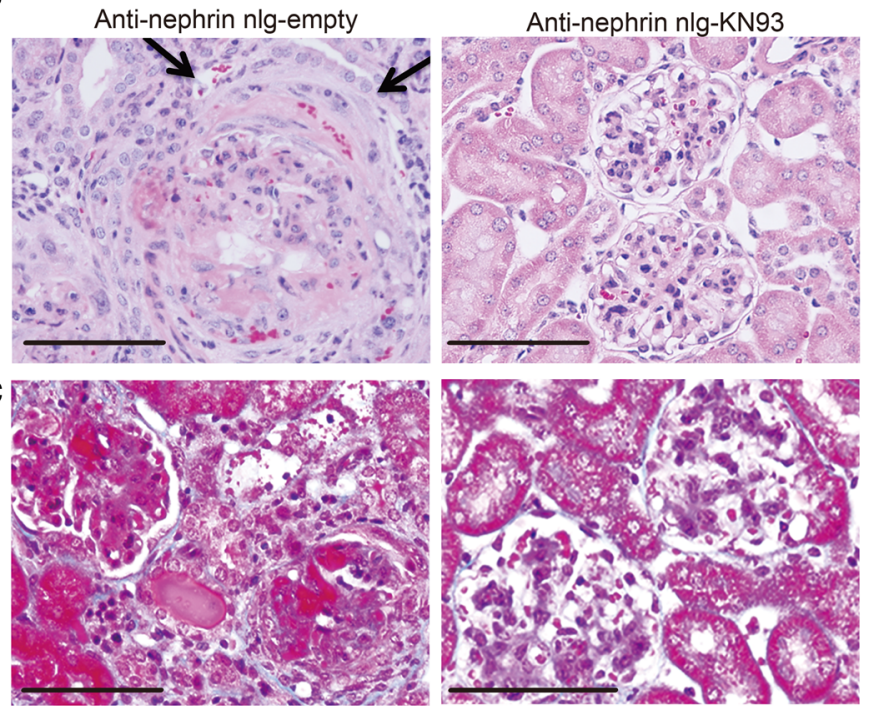

D
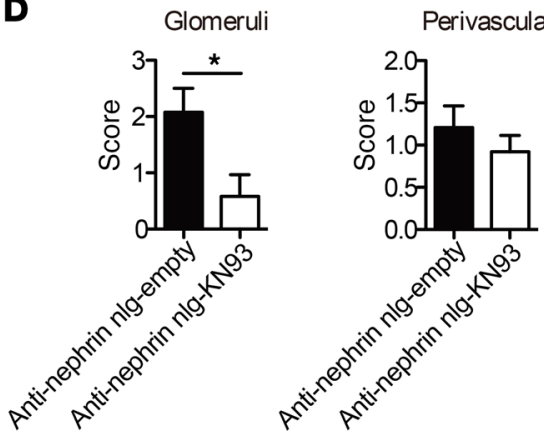

$s^{4}+s^{3}$
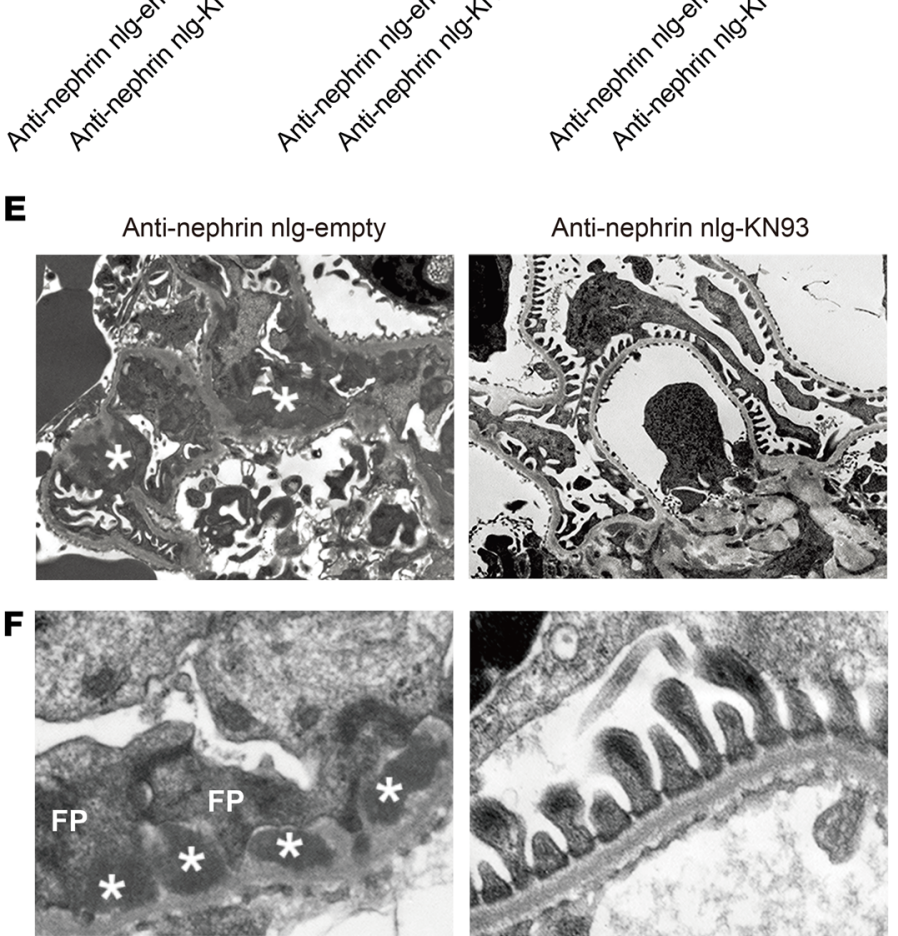

G
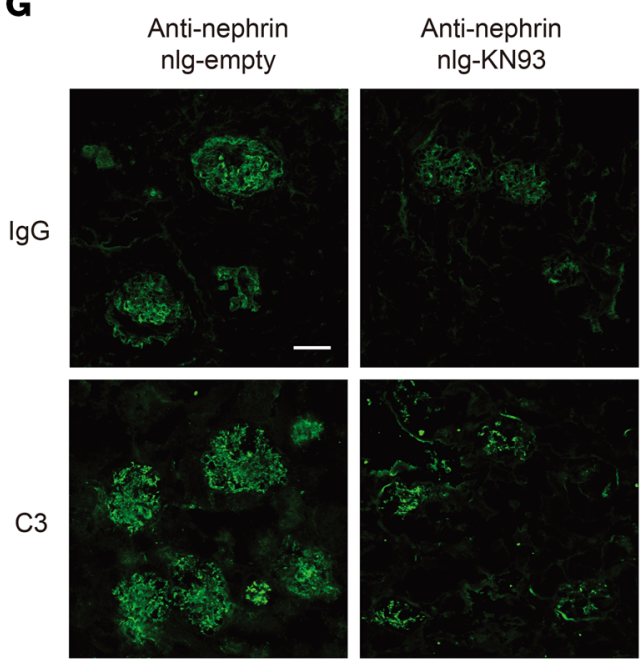

H
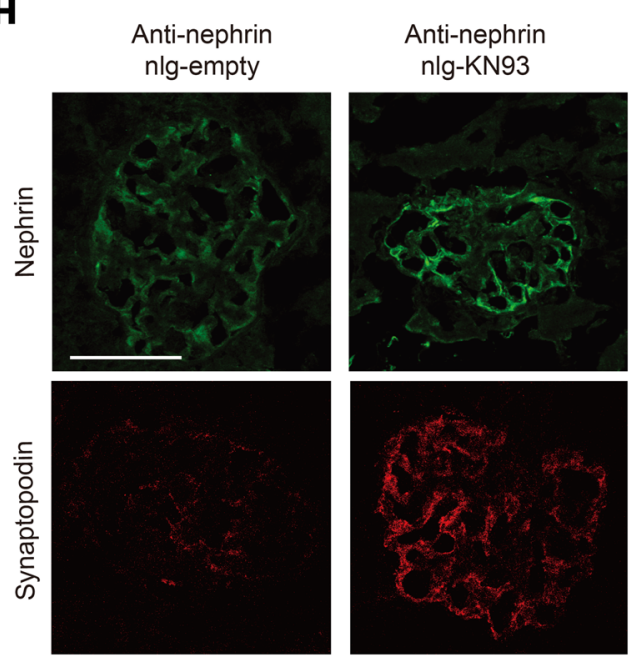

I
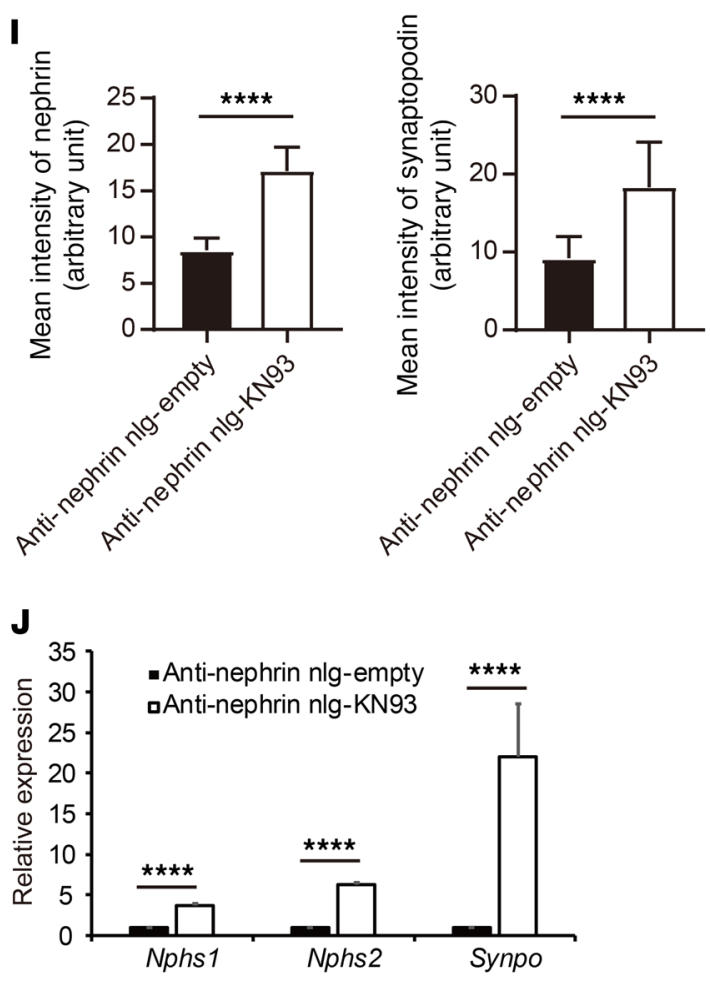
Figure 3. Podocyte-targeted delivery of KN93 suppresses kidney disease development in lupus-prone mice. MRL.Ipr mice were injected i.p. with free KN93 $(10 \mu \mathrm{g} / \mathrm{wk})$, anti-podocin or anti-nephrin antibody-coated KN93loaded nlg (10 $\mu$ g of KN93/week), or empty nlg at from 8 to 16 weeks of age ( $n=5-7$ mice in each group). (A) Urine albumin/creatinine (Alb/Cre) ratio. Urine samples were obtained biweekly, and albumin and creatinine levels were determined by ELISA. Error bars represent mean \pm SEM. ${ }^{*} P<0.05$, 2-way ANOVA with Bonferroni's post test. (B and $\mathbf{C}$ ) Representative images of glomeruli from 16-week- old MRL.Ipr mice treated with anti-nephrin antibody-coated empty nlg or KN93-loaded nlg. PAS (B) and Masson's trichrome staining $(\mathbf{C})$ are shown. The arrows point to the crescent. Scale bars: $50 \mu \mathrm{m}$. (D) Mean histological scores in the kidneys of mice from the indicated treatment groups ( $n=5$ in each group). ${ }^{*} P<0.05$; ${ }^{*} P<0.01$, Student's $t$ test. (E and $\mathbf{F}$ ) Electron microscopic images of a glomerulus from a mouse treated with anti-nephrin empty nlg (left) showing diffuse podocyte foot process (FP) effacement with slit diaphragm occlusion (arrows) and subepithelial dense deposits (asterisks) and a mouse treated with antinephrin KN93-loaded nlg (right), which shows podocytes with normal foot processes and slit diaphragms and no deposits in the basement membrane. Original magnification, $\times 8,000(\mathbf{E}) ; \times 30,000(\mathbf{F})$. Three glomeruli were evaluated in each of 3 mice in each experimental condition. (C) C3 and IgC deposition was significantly suppressed in the glomeruli of MRL.Ipr mice treated with KN93 targeted to podocytes, as indicated. Kidney sections from MRL/ MpJ (16 weeks of age) mice are shown as controls ( $n=5$ mice in each group). (H) Nephrin (green) and synaptopodin (red) expression detected by immunofluorescence. Scale bars: $50 \mu \mathrm{m}$. (I) The fluorescence intensity of nephrin and synaptopodin quantified in glomeruli from mice subjected to the indicated treatments. ${ }^{* * *} P<0.0001$, Student's $t$ test. (J) Nphs1, Nphs2, and Synpo expression in glomeruli from mice treated with anti-nephrin-coated empty or KN93-loaded nlg. Results were normalized to the expression of GAPDH ( $n=5$ in each group). ${ }^{* * *} P<0.0001$, Student's $t$ test.

Figure 11). These data indicate that CaMK4 promotes podocyte motility and remodeling of the podocyte actin cytoskeleton by suppressing synaptopodin expression.

CaMK4 phosphorylates $14-3-3 \beta$ and disrupts its binding to synaptopodin. Next, we sought to elucidate the mechanism whereby activated CaMK4 promotes the degradation of synaptopodin, leading to actin cytoskeleton rearrangement. CaMK4 is activated by binding to $\mathrm{Ca}^{2+} /$ calmodulin, autophosphorylation at multiple Ser residues, and phosphorylation of Thr-200 in its activation loop by the upstream kinase CaMKK (28). The phosphorylation of CaMK4 at Thr-200 has been shown to be fast and transient in response to ionomycin (29). As previously reported, phosphorylated synaptopodin is stabilized though binding with $14-3-3 \beta$ and is released and degraded when dephosphorylated by calcineurin (8). Alternatively, 14-3-3 binding can be disrupted by direct phosphorylation of 14-3-3 (30). We hypothesized that activated CaMK4 disrupts the synaptopodin/14-3-3 $\beta$ complex by phosphorylating 14-3-3 $\beta$. To test this hypothesis, we performed cotransfection studies with 14-3-3ß-FLAG, CAMK4-DsRed, and synaptopodin-6His plasmids, followed by immunoprecipitation with an anti-FLAG or anti-6His antibodies. In these experiments, we treated the cells with ionomycin to activate CaMK4. We observed that phosphoCaMK4 (Thr-200) was increased after ionomycin treatment and interacted with 14-3-3 $\beta$ and not with synaptopodin (Figure 9, A and B). Further, we showed that activated CaMK4 promoted Ser phosphorylation of $14-3-3 \beta$ and that this disrupted its binding to synaptopodin (Figure 9C). Similar data were obtained when we used His-tagged synaptopodin vector (Supplemental Figure 12). These data demonstrate that CaMK4 suppresses synaptopodin expression by enabling its release from the phosphorylated 14-3-3 scaffold protein (Figure 9D).

\section{Discussion}

Our study shows, for what we believe is the first time, that CAMK4 signaling constitutes a common pathway of podocyte dysfunction that leads to proteinuria in autoimmune and nonautoimmune glomerulopathies. Specifically, we provide evidence that CaMK4 is upregulated in podocytes from patients with LN and FSGS as well as in podocytes from animals exposed to LPS or adriamycin. We show that upregulation of CaMK4 represses expression of nephrin, increases the expression of active Rac1 and the motility of podocytes, and phosphorylates the scaffold protein 14-3-3, which results in release and degradation of synaptopodin. More importantly, targeted delivery of a CaMK4 inhibitor obviates the development of LN, as demonstrated by the preservation of podocyte foot processes, absence of immune complex deposition, and formation of crescents in the kidney, without affecting the main elements of systemic autoimmunity. Similarly, targeted inhibition of CaMK4 in podocytes suppresses LPS and adriamycin-induced podocyte damage.

Intravital 2-photon imaging of the 3D structure of mouse podocytes has shown that podocytes remain immobile during normal conditions (31), but if they are injured after exposure to adriamycin or unilateral ureteral ligation, a dynamic podocyte movement is observed (32). Constitutively active Rac1 changes the shape of podocytes, with rapid rearrangement of short lamellipodia-like protrusions, and increases podocyte membrane dynamics in vivo (31). Previous studies have shown that Rac1 activation is not required for normal podocyte function, but active Rac1 induces foot process effacement and rapid onset of proteinuria, which is consistent with a role of Rac1 at the onset of glomerular diseases (33, 34). A mutant form of Arhgap24, a RhoA-activated Rac1 GTPaseactivating protein, suppresses Rac1 and is associated with a familial form of FSGS (35). Our data clearly demonstrate that CaMK4 enhances the expression of activated Rac1, whereas it suppresses the expression of activated RhoA while increasing the motility of podocytes. Silencing of CaMK4 reverses the activation pattern of these 2 GTPases and limits the motility of podocytes. It is interesting to consider the possibility that the suppression of podocyte motility by inhibiting CaMK4 could account for the lack of formation of crescents in the glomeruli of MRL.lpr lupus-prone mice (3).

Previous studies have demonstrated that $\mathrm{Ca}^{2+} /$ calmodulindependent calcineurin signaling plays an important role in regulating podocyte architecture (8). In this study, we show that $\mathrm{Ca}^{2+}$ / CaMK4/synaptopodin signaling is a key driver of podocyte injury that promotes actin rearrangement in podocytes, leading to proteinuria. Src-mediated tyrosine phosphorylation of synaptopodin increases its binding to calcineurin, which in turn dephosphorylates synaptopodin at Ser/Thr residues, which leads to the loss of 14-3-3 binding and the degradation of synaptopodin (36). It would have been expected that the Ser/Thr kinase CaMK4 promotes the phosphorylation of synaptopodin, resulting in stabilization of synaptopodin. However, unexpectedly, we found that inhibition or silencing or genetic deletion of CaMK4 prevented the LPSinduced degradation of synaptopodin and preserved actin structure. Therefore, we focused on the 14-3-3 protein, the chaperone 
A

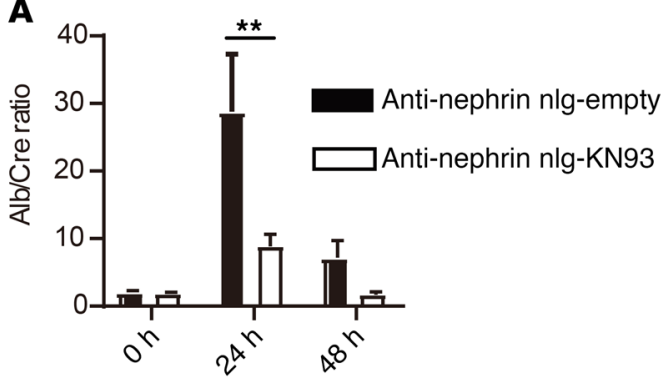

B

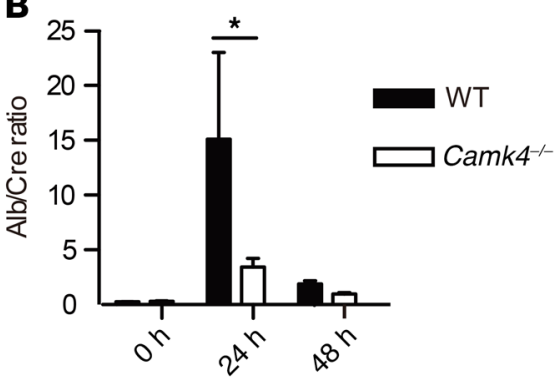

C
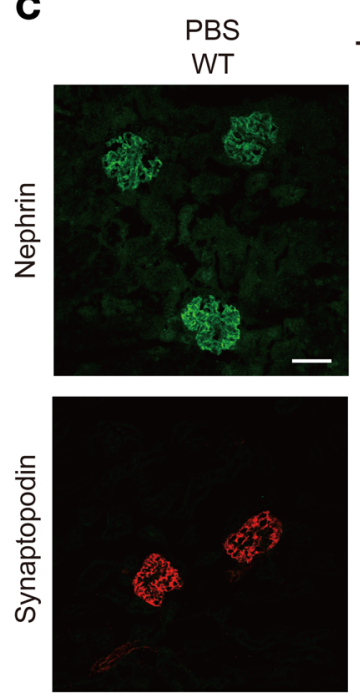

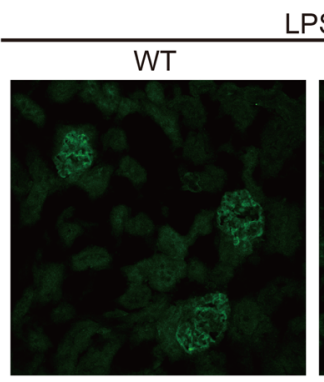

LPS
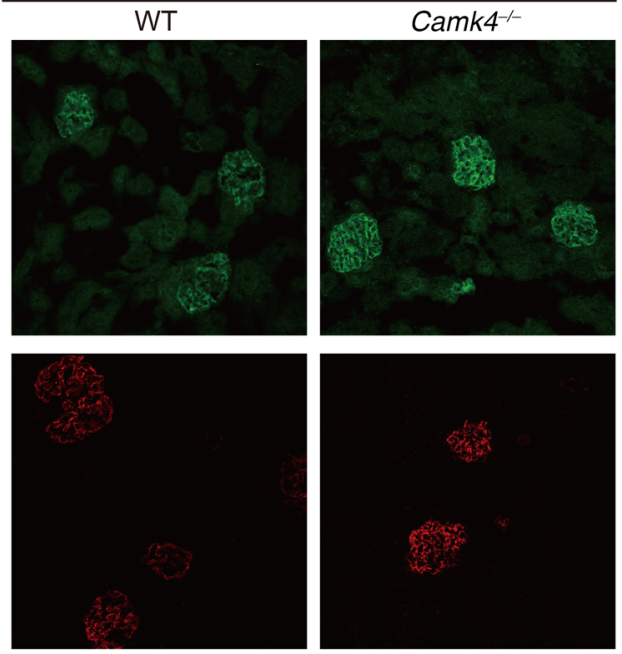

D

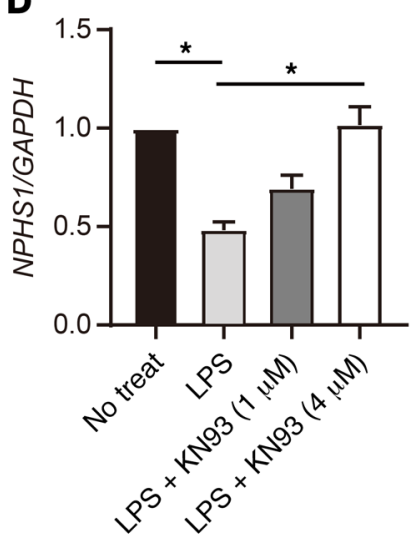

E

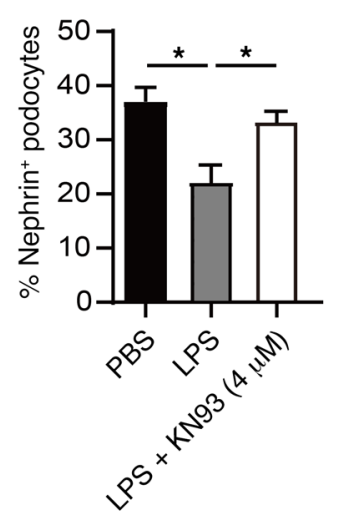

$\mathbf{F}$

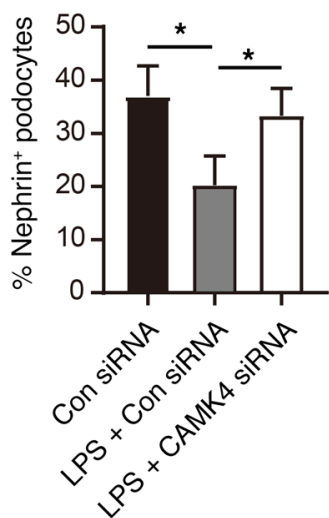

Figure 4. Inhibition or genetic deletion of CaMK4 protects mice from LPS-induced podocyte injury. Mice treated with KN93-loaded nlg targeted to podocytes and CaMK4-deficient mice develop proteinuria after exposure to LPS. Each B6 or B6 Camk4-/- mouse was injected i.p. with LPS on day 0. (A) Urine albumin/creatinine ratio of mice treated with anti-nephrin antibody-coated nlg either empty or loaded with KN93. Nlg were injected i.p. on day -1 ( $n=10$ mice in each group). (B) Urine albumin/creatinine ratio of B6 or B6 Camk4 $4^{-/-}$mice ( $n=6$ in each group). Error bars represent mean $\pm \mathrm{SEM}$. ${ }^{*} P<0.05$; ${ }^{* *} P<0.01$, 2-way ANOVA with Bonferroni's post test. (C) Representative immunofluorescent images of nephrin (upper panels) and synaptopodin (lower panels) in the kidneys of mice injected with LPS or PBS. Scale bar: $50 \mu \mathrm{m}$. (D) Nphs1 expression in human podocytes after stimulation with LPS with or without KN93. Cells were treated with KN93 1 hour before stimulation. Results were normalized by the expression of GAPDH. Four independent experiments were performed. ${ }^{*} P<0.05$, 1-way ANOVA with Tukey's post test. ( $\mathbf{E}$ and $\mathbf{F}$ ) Mean percentage of nephrin-positive human podocytes evaluated by flow cytometry after stimulation with LPS for 72 hours with KN93 (E) or CAMK4 siRNA (F). Four independent experiments were performed. * $P<0.05$, 1-way ANOVA with Tukey's post test.

protein of synaptopodin. The 14-3-3 proteins are phospho-Ser/ phospho-Thr-binding proteins that interact with various binding partners. Indeed, we found that phospho-CaMK4 interacts with and phosphorylates $14-3-3 \beta$, which subsequently displays decreased binding to synaptopodin. This finding documents a function for CaMK4 that involves the phosphorylation of the synaptopodin scaffold protein 14-3-3 and the loss of a protein that is pivotal in the maintenance of podocyte structure and the contractile phenotype of podocytes through the regulation of RhoA and Rac1 activation (21).

Current treatment of autoimmune kidney diseases and nephrotic syndromes, including SLE and FSGS, relies on the use of systemic administration of corticosteroids and cytotoxic drugs, which can cause unwanted side effects. The development of drugs that target molecules involved in the pathogenesis of disease is qualified by the fact that these molecules are expressed by several cell types and tissues and their nonselective blockade or inhibition is invariably associated with side effects. Hence, any pharmacological compound designed to target specific molecules involved in podocyte injury should be delivered specifically and efficiently to podocytes. Our studies offer a treatment modality to circumvent the use of nonspecific immunosuppression in autoimmune and nonautoimmune podocytopathies by delivering an inhibitor of a kinase clearly involved in the loss of podocyte structure and function. The glomerular endothelium and basement membrane are sufficiently perme- 
A

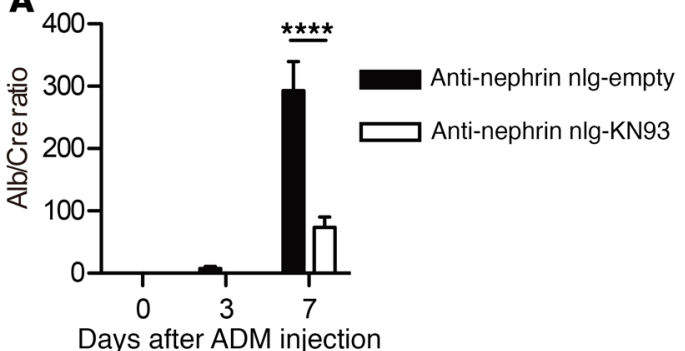

B

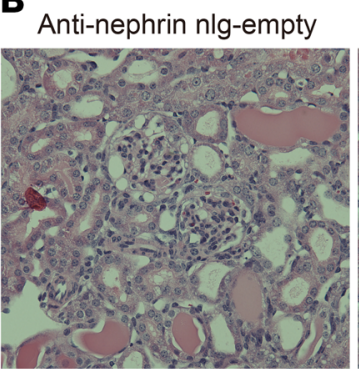

Anti-nephrin nlg-KN93

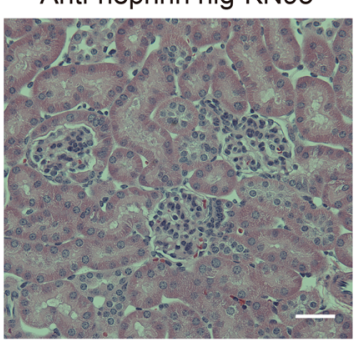

C
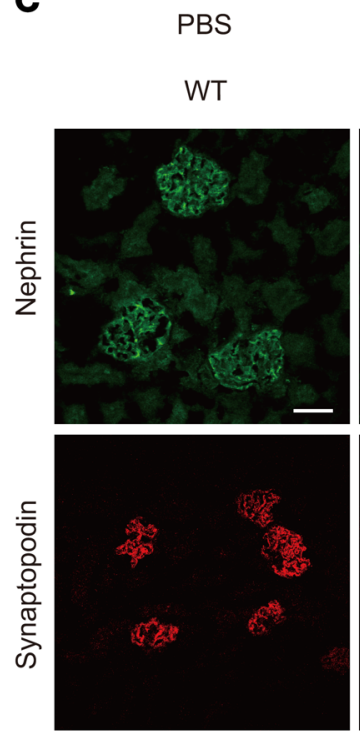

Adriamycin
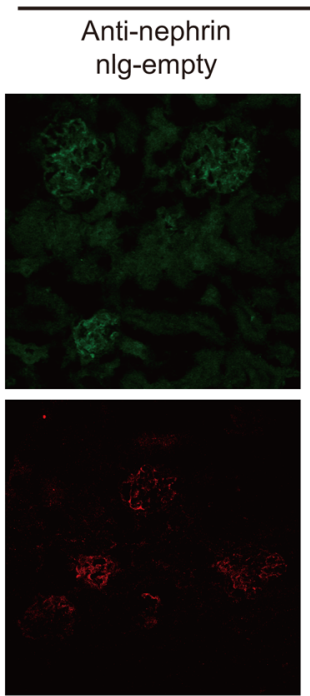

\section{Anti-nephrin} nlg-KN93
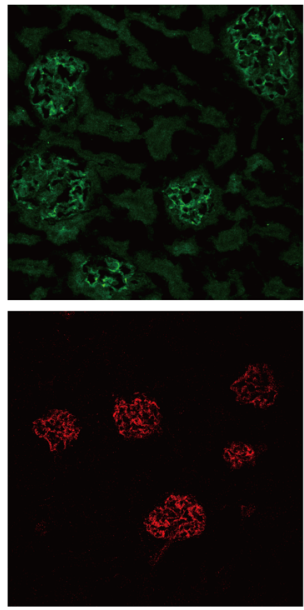

D

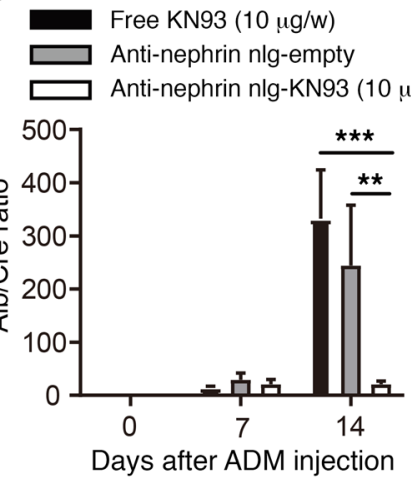

E

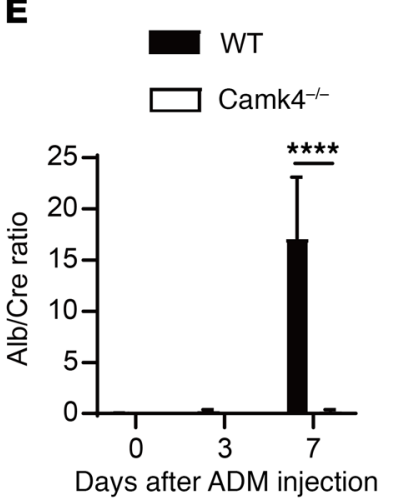

$\mathbf{F}$

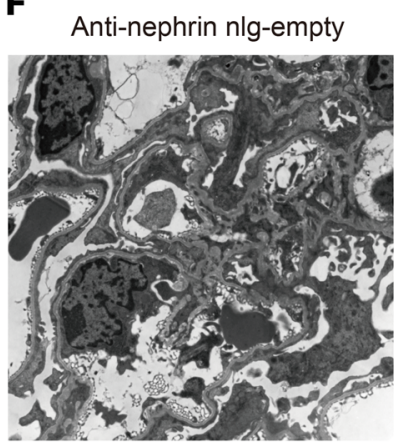

Anti-nephrin nlg-KN93

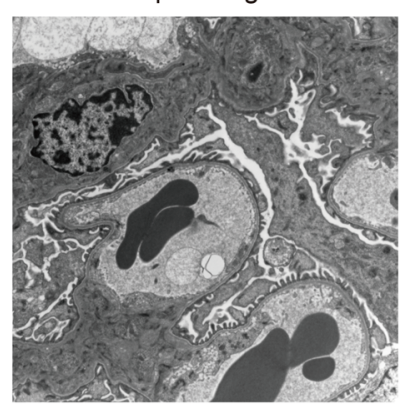

Figure 5. Podocyte-targeted delivery of KN93 prevents and reverses adriamycin-induced podocyte injury in mice. Each mouse was injected i.v. with adriamycin (ADM) on day 0. (A-C) Anti-nephrin antibody-coated empty or KN93-loaded nlg were injected i.p. into BALB/c mice on day -1 and day 3 ( $n=7$ in each group). (A) Mean urine albumin/creatinine ratio from mice subjected to the indicated treatment. Error bars represent mean $\pm S E M$. ${ }^{* * *} P<0.0001$, 2-way ANOVA with Bonferroni's post test (B) Representative images showing PAS staining of kidney from BALB/c mice treated with anti-nephrin antibody-coated empty nlg or KN93-loaded nlg. Scale bar: $50 \mu \mathrm{m}$. (C) Representative immunofluorescence images of nephrin and synaptopodin expression in glomeruli. Scale bar: $100 \mu \mathrm{m}$. (D) Free KN93 $(10 \mu \mathrm{g} / \mathrm{wk})$, anti-nephrin antibody-coated empty, or KN93-loaded nlg (10 $\mu$ g of KN93/wk) were injected i.p. into BALB/c mice on day 7 ( $n=5$ in each group). ${ }^{* *} P<0.01 ;{ }^{* *} P<0.001$, 2-way ANOVA with Bonferroni's post test. (E) Mean urine albumin/creatinine ratio of B6 or B6 Camk4 $4^{--}$mice treated with adriamycin. ( $n=5$ in each group; 2 independent experiments were performed). ${ }^{* * *} P<0.0001,2$-way ANOVA with Bonferroni's post-test. (F) Representative electron microscopy images of glomeruli from BALB/c mice at 7 days after exposure to adriamycin, treated with anti-nephrin antibody-coated empty nlg (left) or KN93-loaded nlg (right). Original magnification, $\times 8,000$.

able for the delivery of targeted nlg to podocytes in vivo, and anti-podocin- and anti-nephrin-coated nlg accumulate in podocytes efficiently compared with noncoated nlg. This targeted delivery accomplishes an equivalent therapeutic effect to doses 10 times larger administered systemically (12).

Podocyte-targeted delivery of the CaMK4 inhibitor had a marked effect on proteinuria in MRL.lpr lupus-prone mice without affecting systemic autoimmunity and the profile of $\mathrm{T}$ cells present in the kidney. We were surprised to observe that this treatment led to a marked reduction of glomerular immune deposits. We had shown before that IgG from patients with LN utilizes the FcRn to enter podocytes and cause increased CaMK4 expression and nephrin loss through not-well-understood pathways (15). The reported increased expression of $\mathrm{FcRn}$ in podocytes from patients with various types of glomerulonephritis, including LN (37), may facilitate this process. Our studies show that increases in CaMK4 result in compromised structure of podocytes, as judged by decreased actin levels, and function, as judged by loss of nephrin and synaptopodin. This leads to the conclusion that if podocytes can maintain their structure and function when CaMK4 is inhibited, immune complexes do not deposit in the glomeruli and probably home in other tissues, such as the spleen and the liver.

We were similarly impressed with our observation that nonimmune podocyte injury caused by LPS and adriamycin in mice was clearly dependent on CaMK4, which when inhibited in a targeted manner, prevented and reversed kidney damage. Notwithstanding the fact that multiple cellular and molecular pathways are involved in the expression of renal injury in patients with LN 


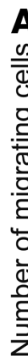

A
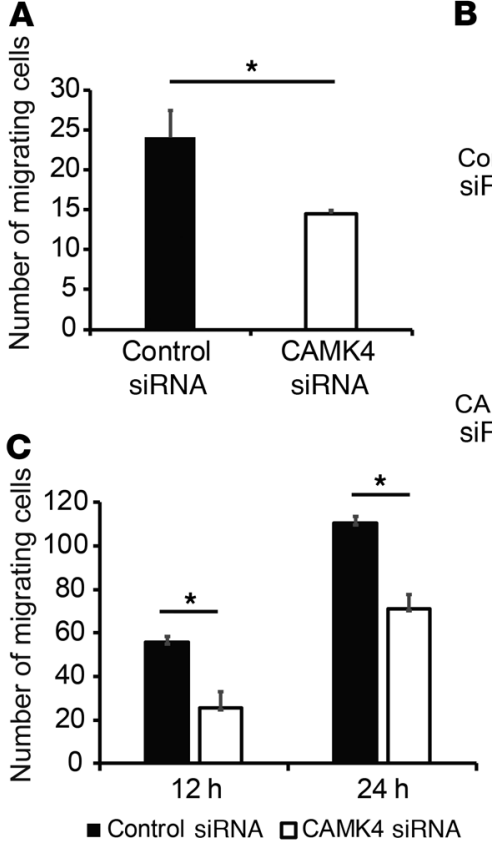

$\mathbf{B}$

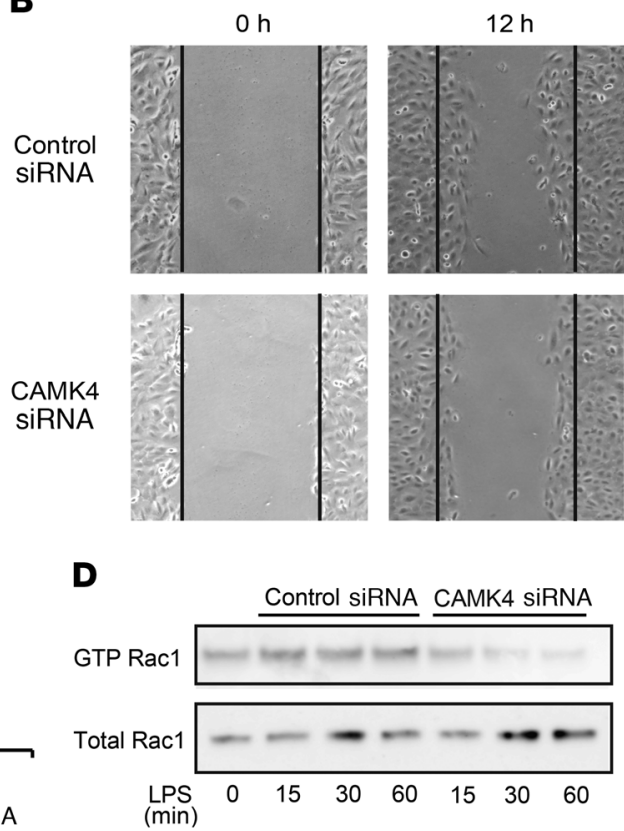

$24 \mathrm{~h}$

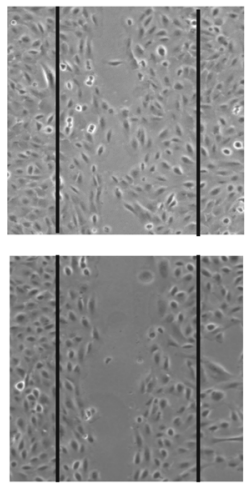

E

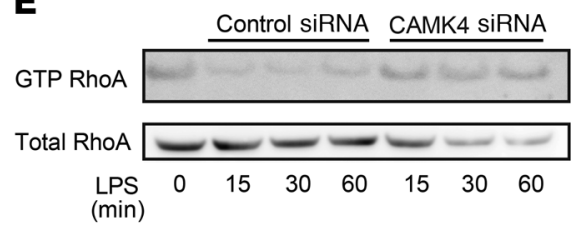

Figure 6. CaMK4 regulates podocyte migration. (A) Result of Transwell migration experiments using human podocytes treated with control or CaMK4 siRNA. Error bars represent mean \pm SEM ( $n=3$ independent experiments). ${ }^{*} P<0.05$, Student's $t$ test. (B) Representative images of wound-healing assay 0,12 , and 24 hours after wounding. (C) Quantification of the results in B. Error bars represent mean $\pm \operatorname{SEM}\left(n=3\right.$ independent experiments). ${ }^{*} P<0.05$, Student's $t$ test. (D and E) Western blotting analysis of GTP-Rac1 and total Rac1 expression (D) and GTP-RhoA and total RhoA (E) in human podocytes treated with control or CaMK4 siRNA for the indicated times. Three independent experiments were performed.

and FSGS, our study demonstrates that CaMK4 is central for the expression of autoimmune and nonautoimmune podocytopathy.

In summary, we reveal CaMK4 as an important Ser/Thr kinase in the control of podocyte structure and function. It is upregulated in human and mouse autoimmune and nonautoimmune glomerular disease and disrupts the expression of nephrin and synaptopodin, the first being involved in the proper function of the slit diaphragm and the second in the maintenance of podocyte actin structure and mobility. Besides contributing to the transcriptional suppression of the nephrin and synaptopodin genes, CaMK4 contributes to the degradation of synaptopodin by causing its release from the scaffold protein 14-3-3 (Figure 9D). Of clinical value is our report that inhibition of CaMK4 solely in podocytes suppresses proteinuria, immune complex deposition, and crescent formation in a lupus-prone mouse. Our finding that CaMK4 is upregulated in the podocytes of patients with FSGS along with the report that CaMK4 inhibitors directed solely to podocytes may prevent and reverse adriamycin-induced disease should have important implications for the treatment of a disease that is a major contributor to the development of CKD and renal failure.

\section{Methods}

Human samples. Human renal biopsies were collected at the Universities of Nagasaki and Nagoya. The patients had biopsy-proven LN $(n=10)$, according to the International Society of Nephrology/Renal Pathology Society criteria (38), or FSGS $(n=10)$. Healthy control samples $(n=6)$ were obtained from Abcam. Clinical data for all patients with LN or FSGS are summarized in Supplemental Table 1.

IgG purification. IgG purification kits (Dojindo Molecular Technologies) were used for purification of IgG from LN patients or healthy controls according to the manufacturer's protocol. Purity was confirmed by SDS-PAGE.

Immortalized human podocyte cell line. The immortalized human podocyte cell line was cultured as previously described (39). Briefly, cells were cultured with RPMI-1640 with $10 \%$ FBS, insulin, transferrin, and selenium. These cells proliferate at $33^{\circ} \mathrm{C}$ and differentiated into mature podocytes for 7 to 10 days after transfer to $37^{\circ} \mathrm{C}$ due to the temperaturesensitive SV40-T gene and a telomerase gene. Ten days after being transferred to $37^{\circ} \mathrm{C}$, cells were treated with LPS $(100 \mu \mathrm{g} / \mathrm{ml})$ or IgG $(50 \mu \mathrm{g} / \mathrm{ml})$ from LN patients or healthy controls. KN93 $(1-4 \mu \mathrm{M})$ or PBS was added 1 hour prior to stimulation. These cells were collected 24 to 72 hours after stimulation and analyzed by flow cytometry or Western blotting. Isolated cells were stained for flow cytometry with antibodies against nephrin (bs-10233R-A488, Bioss Antibodies) and podocin (bs-6597R-A647, Bioss Antibodies). To determine the dead cells in each sample, Zombie Aqua (423102, BioLegend) was added before surface staining.

Mice. Female MRL/MpJ-Tnfrsf6 ${ }^{\text {lpr }}$ (MRL.lpr), female MRL/MPJ, male C57BL/6J (B6), and male BALB/cJ and B6.129X1-Camk4 $4^{\mathrm{tm} 1 \mathrm{Tch}}$ / $\mathrm{J}\left(\mathrm{B} 6 \mathrm{CaMK4} 4^{-/}\right)$mice were purchased from the Jackson Laboratory. MRL.lpr mice were treated with the CaMK4 inhibitor KN93 (Calbiochem), anti-podocin or antinephrin antibody-coated empty, or KN93-loaded nlg once a week from 8 weeks of age. All mice were sacrificed at week 16 .

In adriamycin-induced nephropathy, BALB/cJ, B6, or B6 CaMK4 ${ }^{-1-}$ mice were injected i.v. with adriamycin $(10.0 \mathrm{mg} / \mathrm{kg} / \mathrm{mouse}$ for BALB $/ \mathrm{c}$ or $25 \mathrm{mg} / \mathrm{kg} / \mathrm{mouse}$ for B6 mice) (Selleckchem). Urine samples were collected on days $0,3,7$, or 14, and kidney samples were collected on day 7 or 14 after adriamycin injection.

In the LPS-induced podocyte injury experiments, B6 or B6 $\mathrm{CaMK}^{-/-}$mice were injected i.p. with LPS (200 $\left.\mu \mathrm{g} / \mathrm{mouse}\right)$ (Sigma- 
A
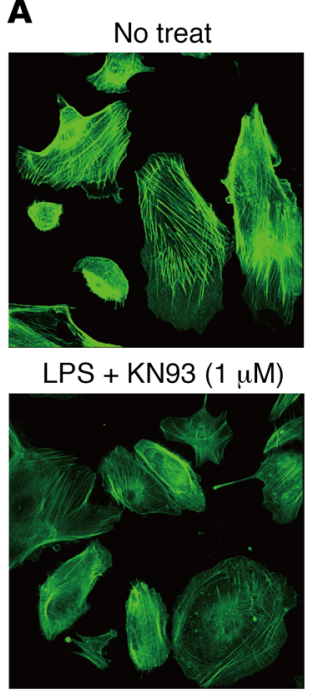

c

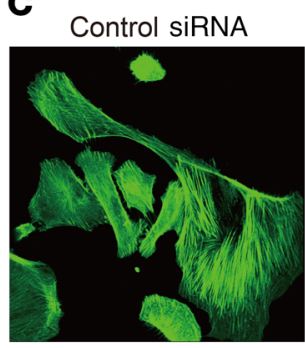

LPS + control siRNA

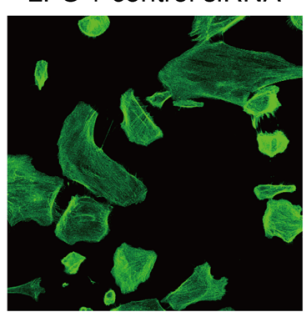

LPS

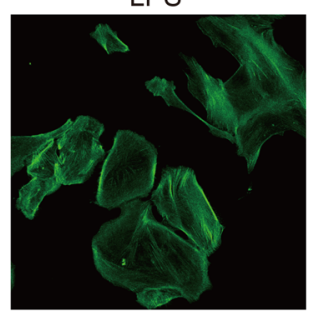

LPS + KN93 (4 $\mu \mathrm{M})$
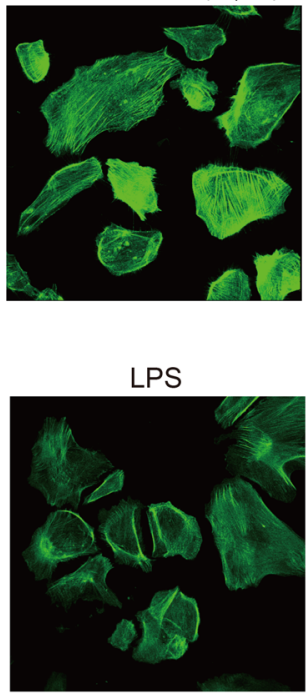

LPS + CAMK4 SiRNA

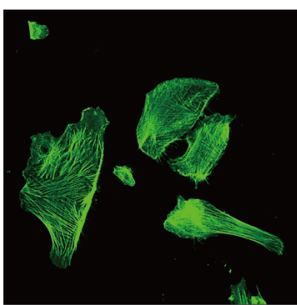

B

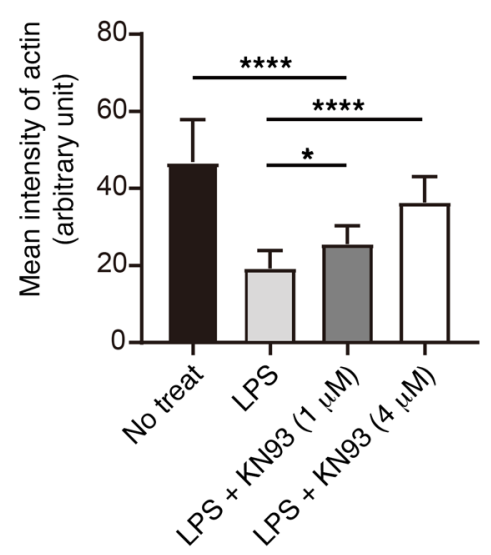

D

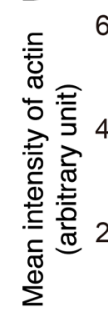

Figure 7. Inhibition or silencing of CaMK4 preserves actin structure of podocytes. (A) Representative results of phalloidin staining of human podocytes after LPS treatment with or without KN93 $(1 \mu \mathrm{M}$ or $4 \mu \mathrm{M})$. (B) Quantification of the results in $\mathbf{A}(n=20$ cells; 3 independent experiments were performed). Error bars represent mean \pm SEM. ${ }^{*} P<0.05 ;{ }^{* * * *} P$ $<0.0001,1$-way ANOVA with Tukey's post test. (C) Representative results of phalloidin staining of human podocytes after LPS treatment with transfection of control or CaMK4 siRNA. (D) Quantification of the results in C $(n=20$ cells; 3 independent experiments were performed). Error bars represent mean \pm SEM. ${ }^{* * *} P<0.0001$, 1-way ANOVA with Tukey's post test. Original magnification, $\times 200$.
Aldrich; E. coli 055: B5). Urine samples were collected at 0, 24, and 48 hours, and kidneys were sampled 24 hours after LPS injection.

Mice were maintained in a specific pathogen-free animal facility (Beth Israel Deaconess Medical Center).

Antibody-coated nlg preparation. KN93-loaded nlg were made as described previously (14). To achieve targeting ability for podocytes, nlg was incubated with biotin-conjugated anti-mouse podocin (bs6597R-Biotin, Bioss Antibodies) or nephrin antibodies (bs-10233RBiotin, Bioss Antibodies) for 20 minutes at room temperature before injection. For analyzing the distribution of nlg after injection in vivo, anti-mouse podocin or nephrin-coated rhodamine B-labeled nlg were injected i.v. into MRL.lpr mice (12 weeks of age), which were euthanized 30 minutes later. The localization of nlg was detected by Nikon Eclipse Ti confocal microscopy.

Western blotting. Podocytes and HEK293T cells were lysed in RIPA buffer or NP4O lysis buffer at $4^{\circ} \mathrm{C}$ for 30 minutes. After centrifugation $\left(16,400 \mathrm{~g} ; 30\right.$ minutes; $\left.4^{\circ} \mathrm{C}\right)$, supernatants were collected and an identical amount of protein from each lysate was separated on NuPAGE 4\%-12\% Bis-Tris Gel (Thermo Fisher). Proteins were transferred to a nitrocellulose membrane, which was subsequently blocked for 1 hour using $5 \%$ nonfat dry milk or $3 \%$ BSA in TBS-T and incubated at $4^{\circ} \mathrm{C}$ overnight with mouse anti-human/mouse CaMK4 (catalog 610276/ clone 26, BD Biosciences), mouse anti-human/mouse CaMK2 (catalog 611293/clone 45, BD Biosciences), goat anti-human/mouse synaptopodin (catalog sc-21537, Santa Cruz Biotechnology Inc.), mouse antihuman GAPDH (catalog 649202/FF26A/F9, BD Biosciences), mouse anti-RhoA (catalog STA-403-A, Cell Biolabs), mouse anti-Rac1 (cata$\log$ STA-401-1, Cell Biolabs), sheep anti-TRPC5 (catalog OST00054G, Thermo Fisher), rabbit anti-FLAG (catalog F7425, MilliporeSigma), mouse anti-phospho-Thr196/200 CaMK4 (provided by Naohito Nozaki, Monoclonal Antibody (MAB) Institute Inc., Iida, Japan), mouse anti-phospho-serine (catalog 612546, BD Biosciences), and mouse anti-14-3-3 (catalog sc-25276/A-6, Santa Cruz Biotechnology Inc.) antibodies. The membrane was washed with TBS-T and incubated with antirabbit or anti-goat, anti-mouse, or anti-sheep IgG coupled with HRP (catalog sc-2004/sc-2020/sc-2005/sc-2473, Santa Cruz Biotechnology Inc.) antibody or mouse anti-rabbit IgG (conformation specific) with HRP antibody (catalog 5127S/L27A9, Cell Signaling Technology). The ECL system (Amersham) was used for detection. Bands on blots corresponding to proteins of interest were analyzed by ImageJ software (NIH). 
A
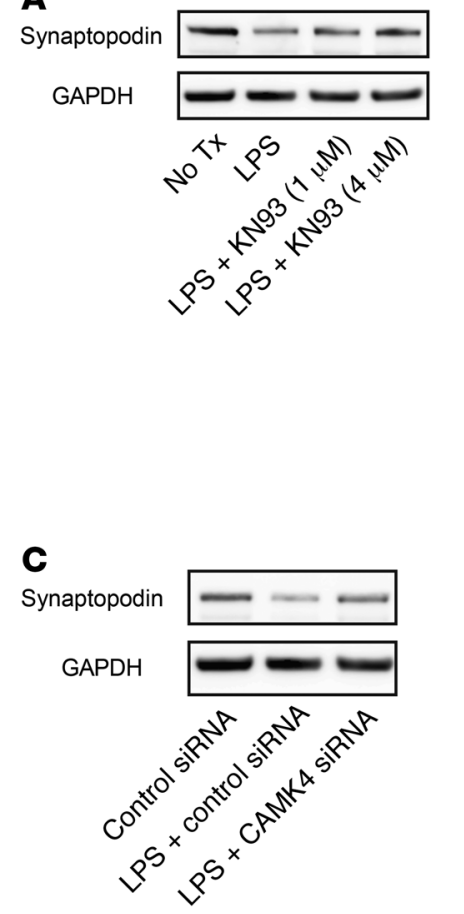
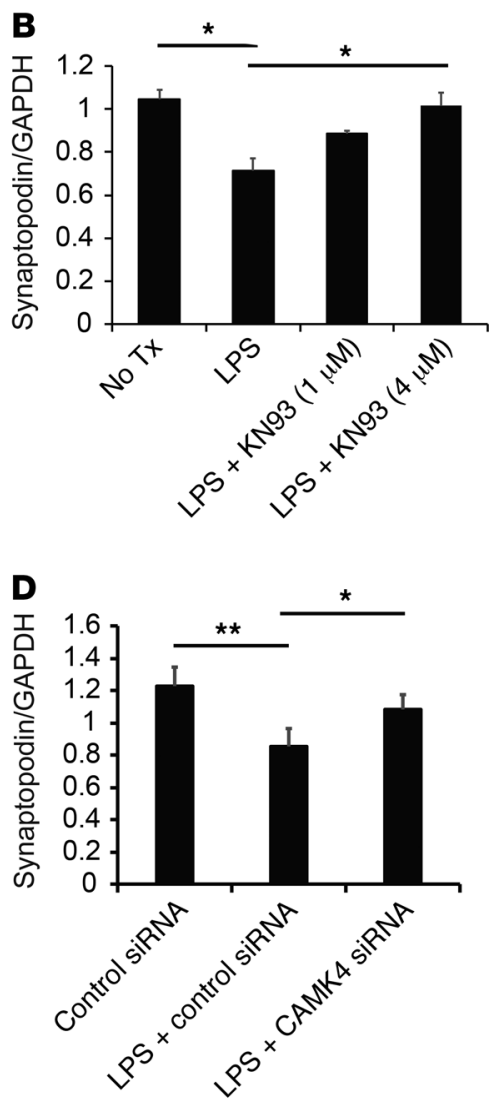

Figure 8. Inhibition or silencing of CaMK4 preserves synaptopodin expression. (A) Western blotting analysis of synaptopodin expression in human podocytes 72 hours after exposure to LPS with or without KN93 $(1 \mu \mathrm{M}$ or $4 \mu \mathrm{M})$. Tx, treatment. (B) Quantification of the results in A. (C) Western blotting analysis of synaptopodin expression in human podocytes 72 hours after exposure to LPS with transfection of control or CaMK4 siRNA. (D) Quantification of the results in C. Error bars represent mean \pm SEM ( $n=3-5$ independent experiments). ${ }^{*} P<0.05 ;{ }^{*} P<0.01$, 1-way ANOVA with Tukey's post-test.

Immunofluorescence. Frozen kidney sections $(4 \mu \mathrm{m})$ were fixed with $4 \%$ formaldehyde for 10 minutes or acetone for 3 minutes and blocked for 1 hour, followed by overnight staining with mouse anti-human/mouse CaMK4 antibody (catalog 610276/clone 26, BD Biosciences), mouse anti-human/mouse CaMK2 antibody (cata$\log 611293 /$ clone 45, BD Biosciences), guinea pig anti-human/ mouse nephrin antibody (catalog GP-N2, Progen), rabbit antimouse CaMK4 antibody (catalog PA1-542, Thermo Fisher), goat anti-human/mouse synaptopodin antibody (catalog sc-21537, Santa Cruz Biotechnology Inc.), goat anti-mouse C3 antibodies conjugated with FITC (catalog 0855510, MP Biomedicals), or goat anti-mouse IgG antibody conjugated with Alexa Fluor 488 (catalog A21206, Invitrogen). Next, sections were stained for 1 hour with Alexa Fluor 488- or 568-labeled goat anti-guinea pig, rabbit, mouse IgG (catalog ab150185/ab175695/ab175701, Abcam) or Alexa Fluor 488- or 568-labeled donkey anti-goat, rabbit, mouse IgG (catalog A21432/ A21202/A21206, Invitrogen). Finally, DAPI or Hoechst 33258 (Life Technologies) was applied for nuclear staining. Stained specimens were analyzed with a Nikon Eclipse Ti confocal microscope. Images were analyzed with EZ-C1 v.3.7 software, and fluorescence intensities or areas were measured by ImageJ software.
Isolation of glomeruli and sorting by flow cytometry. Primary mouse glomeruli were harvested using magnetic isolation protocols. Briefly, mice that had received Dynabead M-450 (Thermo Fisher) perfusions were euthanized, and their kidneys were dissected and digested by type 1 collagenase (Worthington Biochemical) for 30 minutes at $37^{\circ} \mathrm{C}$, then passed through a $100 \mu \mathrm{m}$ filter. Dynabead-trapped glomeruli were subjected to magnetic isolation and washing with PBS. Isolated glomeruli were digested by type- 4 collagenase (Worthington Biochemical) for 15 minutes at $37^{\circ} \mathrm{C}$, and isolated cells were stained for flow cytometry with antibodies against CD45 (catalog 103131/30-F11, BioLegend), CD31 (catalog 102409/MEC13.3, BioLegend), and nephrin (catalog bs-10233R-A488, Bioss Antibodies). To determine the dead cells in each sample, Zombie Aqua (423102, BioLegend) was added before surface staining. Nephrin ${ }^{+} \mathrm{CD} 45^{-} \mathrm{CD} 31^{-}$cells were sorted by BD FACSAria II.

Real-time PCR. Total mRNA was isolated from human podocytes, isolated glomeruli, or sorted podocytes using the RNeasy Mini Kit (QIAGEN), and then cDNA was synthesized using cDNA EcoDry Premix (Clontech) for PCR amplification. Real-time PCR analysis was performed with the Light Cycler 480 System (Roche) using TaqMan gene expression assays according to the manufacturer's specifications (Applied Biosystems). Expression was normalized to GAPDH. All primers and probes were from Applied Biosystems and were as follows: Camk4 (Mm01135329 m1), Nphs1 (Mm01337569_m1), Nphs2 (Mm00450960_m1), Synpo (Mm00441144_g1), Gapdh (Mm99999915_g1), NPHS1 (HsO0190446_m1), and GAPDH (Hs02786624_g1). Gene expression was assessed by the comparative Ct method.

ELISA. Levels of mouse IL-17A/F and IL-2 in serum were measured using ELISA kits (BioLegend and R\&D Systems, respectively). Serum anti-dsDNA antibody concentration was detected using the mouse anti-dsDNA IgG ELISA kit (Alpha Diagnostic International Inc.). Urine albumin and creatinine were quantified using ELISA kits (Bethyl Laboratories and R\&D Systems, respectively).

Flow cytometry. Spleen and lymph nodes were excised from mice, and single-cell suspensions were obtained by teasing the organs through a nylon mesh. Perfused kidneys with PBS were digested with collagenase type $4(100 \mu \mathrm{g} / \mathrm{ml})$ (Worthington Biochemical) in HBSS for 30 minutes $\left(37^{\circ} \mathrm{C}\right)$ to isolate single cells. Isolated cells were stained for flow cytometry with antibodies against CD4 (catalog 100406/ clone GK1.5, BioLegend), CD8a (catalog 100711/clone 53-6.7, BioLegend), CD25 (catalog 102034/clone PC61, BioLegend), CD44 (catalog 103027/clone IM7, BioLegend), CD45 (catalog 103115/clone 30-F11, BioLegend), CD62L (catalog 104408/clone MEL-14, BioLegend) or CD90.2 (catalog 140310/clone 53-2.1, BioLegend) for 30 minutes at $4^{\circ} \mathrm{C}$. To determine the dead cells in each sample, 7 AAD (420404/ BioLegend) was added before applying flow cytometry. Total cell numbers were determined by counting live cells. Absolute cell numbers were calculated on the basis of the percentage of each population. For intracellular cytokine staining, cells were isolated as described above and were stimulated for 4 hours in culture medium containing PMA ( $5 \mathrm{ng} / \mathrm{ml}$; Sigma-Aldrich), ionomycin ( $500 \mathrm{ng} / \mathrm{ml}$; Sigma-Aldrich), and monensin (GolgiStop; BD Biosciences). To detect the dead cells in each sample, Zombie Aqua dye (423102, BioLegend) was added and cells were washed twice with PBS. Then, after staining of surface markers 
A

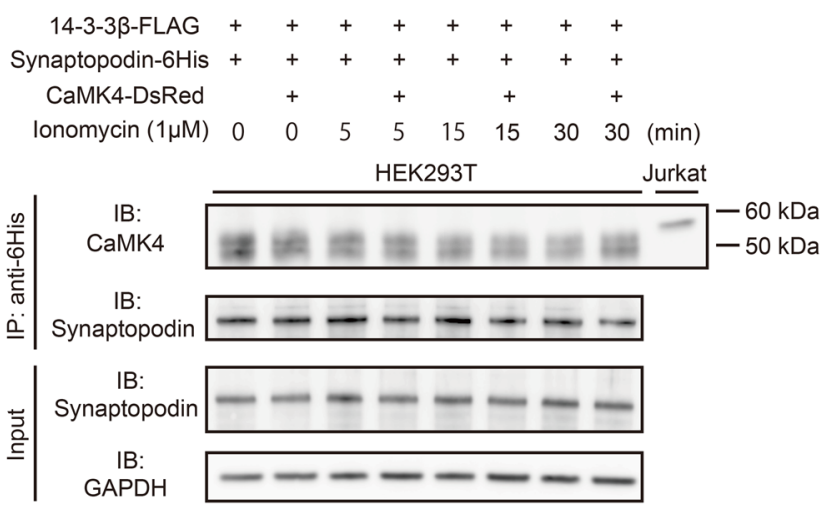

C

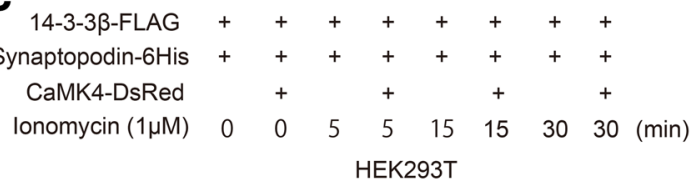

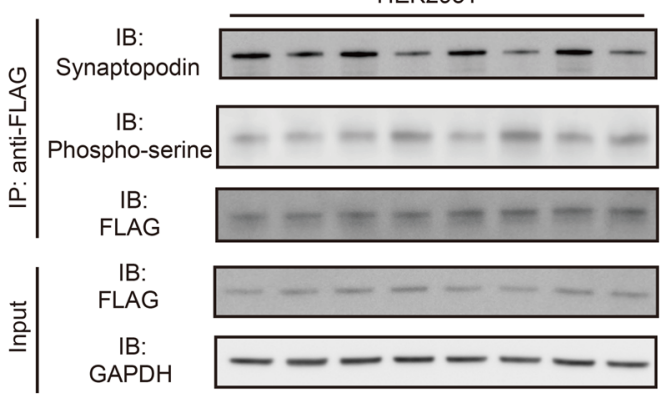

B
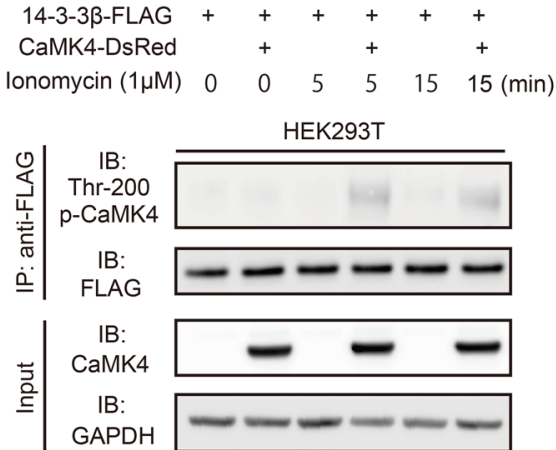

D

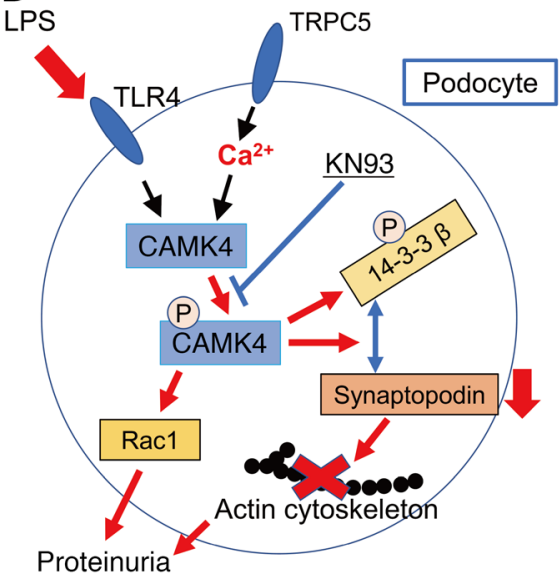

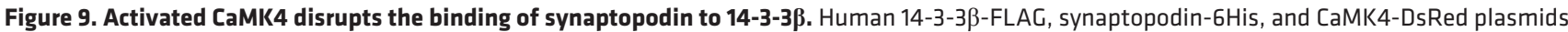
are transfected into HEK293T cells, followed by immunoprecipitation with an anti-FLAG or anti-6His antibodies. (A) Immunoprecipitation with anti-6His antibody and immunoblot analysis of CaMK4 and synaptopodin. CaMK4 does not interact with His-tagged synaptopodin in cotransfected HEK293T cells. Jurkat cell lysate was used as a positive control of CaMK4. Data are representative of 2 independent experiments. (B) Phospho-CaMK4 precipitates with flag-tagged 14-3-3 $\beta$ after exposure to ionomycin from cotransfected HEK293T cells. Data are representative of 2 independent experiments. (C) Immunoprecipitation and immunoblot analysis of synaptopodin and phospho-serine expression. Cotransfected HEK293T cells were stimulated with ionomycin for the indicated times. Cell lysates were immunoprecipitated with anti-FLAG antibody and then analyzed by immunoblotting with anti-synaptopodin and anti-phospho-serine antibodies. Data are representative of 3 independent experiments. (D) Schematic model of the relationship between $\mathrm{Ca}^{2+} / \mathrm{CaMK} \mathrm{M}$ signaling and actin stability in podocytes.

CD4 (100406/GK1.5, BioLegend), CD8a (100713/53-6.7, BioLegend), CD45 (102409/30-F11, BioLegend), or CD90.2 (140310/53-2.1, BioLegend) for 30 minutes at $4^{\circ} \mathrm{C}$, cells were fixed and made permeable with Cytofix/Cytoperm and Perm/Wash buffer according to the manufacturer's instructions (BD Biosciences). Then cells were stained with antibodies to cytokines (anti-IFN- $\gamma$; 505830/XMG1.2 or antiIL-17A; 506904/TC11-18H10.1, BioLegend). For FOXP3 staining, cells were permeabilized using the Mouse Regulatory T cell Staining Kit (eBioscience) and anti-FOXP3 antibody (catalog 12-5773-82/ FJK-16s, eBioscience). All flow cytometry data were acquired on a BD LSRII and analyzed with FlowJo software.

Periodic acid-Schiff and Masson's trichrome staining. Kidneys were fixed in $10 \%$ formalin, paraffin sections were stained with periodic acid-Schiff (PAS) or Masson's trichrome, and renal lesions were evaluated according to previously described criteria (12). Histological scoring for kidney samples was performed blindly by a nephropathologist.

Electron microscopy. For ultrastructural examination, $1 \mathrm{~mm}^{3}$ pieces of randomly selected renal cortical tissue from mice in control and experimental groups were fixed in 2.5\% PBS-buffered glutaraldehyde, postfixed in $1 \%$ osmium tetroxide, dehydrated in ethanol, and embedded in Spurr's epoxy resin. Toluidine blue-stained semithin sections were examined for the presence of glomeruli. Ultrathin sections were stained with uranyl acetate and lead citrate and examined with a Phillips CM10 electron microscope.

Transwell migration assay. Transwell cell culture inserts (pore size, $5 \mu \mathrm{m}$; Costar Corp., Corning) were coated with type I collagen, rinsed once with PBS, and placed in RPMI medium in the lower compartment. For each experiment, $2 \times 10^{4}$ control and CaMK4 knockdown podocytes were seeded in the inserts and allowed to migrate for 24 hours while being incubated at $37^{\circ} \mathrm{C}$. Nonmigratory cells were removed from the upper surface of the membrane, and migrated cells were fixed with cold methanol and stained with Crystal Violet Solution (MilliporeSigma). The number of migrated cells was counted with a $\times 20$ objective in the center of a membrane (1 field).

Wound-healing assay. Control and CaMK4-knockdown podocytes $\left(\right.$ each $\left.8 \times 10^{5}\right)$ were seeded overnight on type I collagen-coated cover- 
slips in 6-well plates. Each well was then scratched with a sterile $200 \mu \mathrm{l}$ pipette tip, washed with PBS, and placed into fresh medium. Pictures were captured by phase-contrast microscopy under a $\times 10$ objective at 12 and 24 hours after scratching, and the number of cells that had migrated into the same-sized square fields were counted.

Rac1 and RhoA activation assay. Podocytes were treated with LPS $(100 \mu \mathrm{g} / \mathrm{ml})$ for the indicated time period, followed by harvest, and Rac1 or RhoA pulldown experiments were performed. In the KN93 experiments, cells were pretreated with $4 \mu \mathrm{M}$ KN93 for 1 hour before LPS was applied. Activated Rac1 or RhoA was analyzed with a commercial Rac1 or RhoA activation assay kit (Cell Biolabs). The eluted active Rac1 or RhoA was detected by immunoblotting using a mouse monoclonal Rac1 or RhoA antibody (Cell Biolabs). Total Rac1, RhoA, and GAPDH were measured in the cell lysates used for the pulldown studies and served as loading controls.

Phalloidin staining. Cultured podocytes were seeded onto type I collagen 4-well culture slides (Corning). The cells were washed once with PBS and fixed for 20 minutes with $4 \%$ paraformaldehyde. The cells were permeabilized for 5 minutes with $0.1 \%$ Triton X-100 in PBS, followed by blocking for 30 minutes with PBS containing 2\% BSA. Cells were then stained for 1 hour at room temperature with AF488labeled phalloidin (A12379, Thermo Fisher). After washing 3 times with PBS, the stained cover glasses were mounted on a glass slide with Hoechst 33258 and sealed with nail polish. Stained specimens were analyzed with a Nikon Eclipse Ti confocal microscope. Images were analyzed with EZ-C1 v.3.7 software, and fluorescence intensities of actin fibers were measured by ImageJ software.

Transfection of siRNA. Human podocytes were cultured for 7 to 10 days for differentiation and then transfected with CAMK4 siRNA, TRPC5 siRNA, or control siRNA (GE Dharmacon) by Lipofectamine RNAiMAX Transfection Reagent (Thermo Fisher) according to the manufacturer's protocol. After 48 or 72 hours of incubation, the cells were stimulated with LPS or were collected for Transwell migration or wound-healing assay.

Cotransfection and immunoprecipitation. HEK293T cells (ATCC) were cultured with DMEM with $10 \%$ FBS and were transfected with pcDNA-Flag-HA-14-3-3 $\beta$ (Addgene), pcDNA-6His-synaptopodin, pIRES-DsRed-Express-CaMK4, or pIRES-DsRed-Express empty vector (Clontech Laboratories Inc.) using Lipofectamine 3000 (Thermo Fisher). pcDNA-6His-synaptopodin and pIRES-DsRed-ExpressCaMK4 were made by Genescript. Forty-eight hours after transfection, the transfected cells were subjected to stimulation with ionomycin $(1 \mu \mathrm{M})$ for the indicated times. Cells were lysed by NP-40 cell lysis buffer (Thermo Fisher), and the lysate was used to perform immunoprecipitation with anti-FLAG antibody (F7425, MilliporeSigma) or anti-6His antibody (MA1-135, Thermo Fisher) using the Dynabeads Protein G Immunoprecipitation Kit (Thermo Fisher).

Statistics. Statistical analyses were performed in GraphPad Prism version 7.0 software. Statistical significance was determined by $t$ tests (2-tailed) for 2 groups, 1-way ANOVA with Tukey's multiple comparisons tests, or 2-way ANOVA with Bonferroni's multiple comparisons tests for 3 or more groups. $P<0.05$ was considered statistically significant.

Study approval. Human renal biopsies were collected at the Universities of Nagasaki and Nagoya after informed consent was obtained. The protocol concerning the use of biopsy samples from patients with LN and FSGS was approved by the Institutional Review Boards on human subjects at Nagasaki University Hospital (no. 12012397) and Nagoya University Hospital (no. 1135). All animal protocols and experiments were approved by the Institutional Animal Care and Use Committee of Beth Israel Deaconess Medical Center (no. 088-2015).

\section{Author contributions}

GCT conceived and supervised the overall project, designed experiments, and analyzed results. $\mathrm{KM}$ and $\mathrm{KO}$ designed and performed experiments and analyzed data. NY and MK performed experiments and analyzed data. MSAA and MGT performed electron microscopic experiments and analyzed data. KI, SM, and TN performed human kidney immunohistochemistry and analyzed data. SB and TMF generated nlg and analyzed data. RB and AF analyzed data. KM, KO, MGT, and GCT wrote the manuscript.

\section{Acknowledgments}

We thank Martin Polack of Harvard Medical School for allowing us to use the immortalized human podocyte cell line. We also thank Hallie Lappin for her assistance with the electron microscopy. This work was supported by NIH grants R01AR064350 (to GCT), and T32AIO07512 (to AF), the Lupus Insight Award from the Lupus Research Alliance (to GCT), and NIH Medical Scientist Training Program training grant T32GM007205 (SB).

Address correspondence to: George C. Tsokos, Beth Israel Deaconess Medical Center, Harvard Medical School, 330 Brookline Avenue, CLS-937, Boston, Massachusetts 02215, USA. Phone: 617.735.4160; Email: gtsokos@bidmc.harvard.edu.
1. Breyer MD, Susztak K. The next generation of therapeutics for chronic kidney disease. Nat Rev Drug Discov. 2016;15(8):568-588.

2. D’Agati VD, Kaskel FJ, Falk RJ. Focal segmental glomerulosclerosis. $N$ Engl JMed. 2011;365(25):2398-2411.

3. Scott RP, Quaggin SE. Review series: The cell biology of renal filtration. J Cell Biol. 2015;209(2):199-210.

4. Welsh GI, Saleem MA. The podocyte cytoskeleton-key to a functioning glomerulus in health and disease. Nat Rev Nephrol. 2011;8(1):14-21.

5. Perico L, Conti S, Benigni A, Remuzzi G. Podocyte-actin dynamics in health and disease. Nat Rev Nephrol. 2016;12(11):692-710.
6. Greka A, Mundel P. Calcium regulates podocyte actin dynamics. Semin Nephrol. 2012;32(4):319-326.

7. Schaldecker T, et al. Inhibition of the TRPC5 ion channel protects the kidney filter. JClin Invest. 2013;123(12):5298-5309.

8. Faul C, et al. The actin cytoskeleton of kidney podocytes is a direct target of the antiproteinuric effect of cyclosporine A. Nat Med. 2008;14(9):931-938.

9. Jiang L, et al. Calmodulin-dependent protein kinase II/cAMP response element-binding protein/Wnt/ $\beta$-catenin signaling cascade regulates angiotensin II-induced podocyte injury and albuminuria. J Biol Chem. 2013;288(32):23368-23379.
10. Racioppi L, Means AR. Calcium/calmodulindependent kinase IV in immune and inflammatory responses: novel routes for an ancient traveller. Trends Immunol. 2008;29(12):600-607.

11. Koga T, et al. CaMK4-dependent activation of $\mathrm{AKT} / \mathrm{mTOR}$ and CREM- $\alpha$ underlies autoimmunity-associated Th17 imbalance. JClin Invest. 2014;124(5):2234-2245.

12. Ichinose K, Juang YT, Crispín JC, Kis-Toth K, Tsokos GC. Suppression of autoimmunity and organ pathology in lupus-prone mice upon inhibition of calcium/calmodulin-dependent protein kinase type IV. Arthritis Rheum. 2011;63(2):523-529.

13. Koga T, Ichinose K, Mizui M, Crispín JC, Tsokos GC. Calcium/calmodulin-dependent protein 
kinase IV suppresses IL-2 production and regulatory T cell activity in lupus. J Immunol. 2012;189(7):3490-3496.

14. Otomo K, et al. Cutting edge: nanogel-based delivery of an inhibitor of CaMK4 to CD4+ T cells suppresses experimental autoimmune encephalomyelitis and lupus-like disease in mice. J Immunol. 2015;195(12):5533-5537.

15. Ichinose K, et al. Lupus nephritis IgG induction of calcium/calmodulin-dependent protein kinase IV expression in podocytes and alteration of their function. Arthritis Rheumatol. 2016;68(4):944-952.

16. Greka A, Mundel P. Cell biology and pathology of podocytes. Annu Rev Physiol. 2012;74:299-323.

17. Park J, et al. Combination delivery of TGF- $\beta$ inhibitor and IL-2 by nanoscale liposomal polymeric gels enhances tumour immunotherapy. Nat Mater. 2012;11(10):895-905.

18. Thorner PS, Ho M, Eremina V, Sado Y, Quaggin S. Podocytes contribute to the formation of glomerular crescents. J Am Soc Nephrol. 2008;19(3):495-502.

19. Reiser J, et al. Induction of B7-1 in podocytes is associated with nephrotic syndrome. JClin Invest. 2004;113(10):1390-1397.

20. Lee VW, Harris DC. Adriamycin nephropathy: a model of focal segmental glomerulosclerosis. Nephrology (Carlton). 2011;16(1):30-38.

21. Mundel P, Reiser J. Proteinuria: an enzymatic disease of the podocyte? Kidney Int . 2010;77(7):571-580.

22. Kistler AD, Altintas MM, Reiser J. Podocyte
GTPases regulate kidney filter dynamics. Kidney Int. 2012;81(11):1053-1055.

23. Asanuma K, Yanagida-Asanuma E, Faul C, Tomino Y, Kim K, Mundel P. Synaptopodin orchestrates actin organization and cell motility via regulation of RhoA signalling. Nat Cell Biol. 2006;8(5):485-491.

24. Etienne-Manneville S, Hall A. Rho GTPases in cell biology. Nature. 2002;420(6916):629-635.

25. Wang L, et al. Mechanisms of the proteinuria induced by Rho GTPases. Kidney Int . 2012;81(11):1075-1085.

26. Tian X, Ishibe S. Targeting the podocyte cytoskeleton: from pathogenesis to therapy in proteinuric kidney disease. Nephrol Dial Transplant . 2016;31(10):1577-1583.

27. Tian D, et al. Antagonistic regulation of actin dynamics and cell motility by TRPC5 and TRPC6 channels. Sci Signal. 2010;3(145):ra77.

28. Soderling TR. The Ca-calmodulin-dependent protein kinase cascade. Trends Biochem Sci. 1999;24(6):232-236.

29. Dias WB, Cheung WD, Wang Z, Hart GW. Regulation of calcium/calmodulin-dependent kinase IV by O-GlcNAc modification.J Biol Chem. 2009;284(32):21327-21337.

30. Morrison DK. The 14-3-3 proteins: integrators of diverse signaling cues that impact cell fate and cancer development. Trends Cell Biol. 2009;19(1):16-23.

31. Brähler S, et al. Intravital and kidney slice imaging of podocyte membrane dynamics. J Am Soc
Nephrol. 2016;27(11):3285-3290.

32. Hackl MJ, et al. Tracking the fate of glomerular epithelial cells in vivo using serial multiphoton imaging in new mouse models with fluorescent lineage tags. Nat Med.2013;19(12):1661-1666.

33. Blattner SM, et al. Divergent functions of the Rho GTPases Rac1 and Cdc42 in podocyte injury. Kidney Int. 2013;84(5):920-930.

34. $\mathrm{Yu} \mathrm{H}$, et al. Rac1 activation in podocytes induces rapid foot process effacement and proteinuria. Mol Cell Biol. 2013;33(23):4755-4764.

35. Akilesh S, et al. Arhgap24 inactivates Rac1 in mouse podocytes, and a mutant form is associated with familial focal segmental glomerulosclerosis. J Clin Invest. 2011;121(10):4127-4137.

36. Buvall L, et al. Synaptopodin is a coincidence detector of tyrosine versus serine/threonine phosphorylation for the modulation of Rho protein crosstalk in podocytes. J Am Soc Nephrol. 2017;28(3):837-851.

37. Gan H, et al. Neonatal Fc receptor stimulation induces ubiquitin c-terminal hydrolase-1 overexpression in podocytes through activation of p38 mitogen-activated protein kinase. Hum Pathol. 2012;43(9):1482-1490.

38. Weening JJ, et al. The classification of glomerulonephritis in systemic lupus erythematosus revisited. Kidney Int. 2004;65(2):521-530.

39. Saleem MA, et al. A conditionally immortalized human podocyte cell line demonstrating nephrin and podocin expression. JAm Soc Nephrol. 2002;13(3):630-638. 